\title{
Robotic vs. Retropubic radical prostatectomy in prostate cancer: A systematic review and a meta-analysis update
}

\author{
Kun Tang ${ }^{1, *}$, Kehua Jiang ${ }^{1,2, *}$, Hongbo Chen ${ }^{2}$, Zhiqiang Chen ${ }^{1}$, Hua $X_{u^{1}}$ and Zhangqun \\ $\mathbf{Y e}^{1}$ \\ ${ }^{1}$ Department of Urology, Institute of Urology, Tongji Hospital, Tongji Medical College, Huazhong University of Science and \\ Technology, Wuhan, China \\ 2 Department of Urology, The Central Hospital of Enshi Autonomous Prefecture, Enshi, China \\ * These authors have contributed equally to this work
}

Correspondence to: Hua Xu, email: xuhuawhu@163.com

Keywords: robotic-assisted radical prostatectomy; retropubic radical prostatectomy; prostate cancer; meta-analysis; update

Received: February 18, $2016 \quad$ Accepted: October 21, $2016 \quad$ Published: November 12, 2016

Copyright: Tang et al. This is an open-access article distributed under the terms of the Creative Commons Attribution License (CC-BY), which permits unrestricted use, distribution, and reproduction in any medium, provided the original author and source are credited.

\section{ABSTRACT}

CONTEXT: The safety and feasibility of robotic-assisted radical prostatectomy (RARP) compared with retropubic radical prostatectomy(RRP) is debated. Recently, a number of large-scale and high-quality studies have been conducted.

OBJECTIVE: To obtain a more valid assessment, we update the meta-analysis of RARP compared with RRP to assessed its safety and feasibility in treatment of prostate cancer.

METHODS: A systematic search of Medline, Embase, Pubmed, and the Cochrane Library was performed to identify studies that compared RARP with RRP. Outcomes of interest included perioperative, pathologic variables and complications.

RESULTS: 78 studies assessing RARP vs. RRP were included for meta-analysis. Although patients underwent RRP have shorter operative time than RARP (WMD: 39.85 minutes; $P<0.001$ ), patients underwent RARP have less intraoperative blood loss (WMD = $-507.67 \mathrm{ml} ; P<\mathbf{0 . 0 0 1})$, lower blood transfusion rates $(\mathrm{OR}=$ $0.13 ; P<0.001)$, shorter time to remove catheter (WMD = -3.04day; $P<0.001$ ), shorter hospital stay (WMD = -1.62day; $P<0.001$ ), lower PSM rates (OR:0.88; $P=$ $0.04)$, fewer positive lymph nodes (OR:0.45; $P<0.001)$, fewer overall complications (OR:0.43; $P<0.001)$, higher $3-$ and 12 -mo potent recovery rate (OR:3.19; $P=0.02$; OR: 2.37; $P=0.005$, respectively), and lower readmission rate (OR:0.70, $P=0.03)$. The biochemical recurrence free survival of RARP is better than RRP (OR:1.33, P = $0.04)$. All the other calculated results are similar between the two groups.

CONCLUSIONS: Our results indicate that RARP appears to be safe and effective to its counterpart RRP in selected patients.

\section{INTRODUCTION}

Prostate cancer $(\mathrm{PCa})$ is the most common cancer in the worldwide and its morbidity,mortality is the first and second common cancer in men, respectively [1]. RP is the standard therapy for patients with localized PCa [2]. However, open retropubic radical prostatectomy (RRP) is associated with higher overall complications, including estimated blood loss (EBL), wound infections. With the development of surgical techniques, laparoscopic techniques and robot assisted surgeries have become a very popular procedure for the management of urological disease throughout the world [3]. Compared with RRP, the advantages of laparoscopic radical prostatectomy (LRP) are less EBL, fewer complications, better cosmetic effect and shorter hospital stay [4]. The disadvantages of LRP is lack of 3D visualization and poor ergonomics.

As alternatives to open surgery, RARP has became a predominant procedure for the treatment the localized prostate cancer in the world [5]. Assessing of the robotic 
Table 1: Characteristics of included studies

\begin{tabular}{|c|c|c|c|c|c|c|c|}
\hline First author, year & Country & Study interval & Design & LOE & \begin{tabular}{|l|} 
No.of \\
patients \\
RARP/RRP
\end{tabular} & $\begin{array}{l}\text { Matching/ } \\
\text { comparable* }\end{array}$ & Quality score ${ }^{\Delta}$ \\
\hline Ahlering, 2004 & USA & 2001-2002 & Prospective & $3 b$ & $60 / 60$ & $1,2,3,4$ & $* * * * *$ \\
\hline Bae, 2012 & Korea & 2008-2011 & Retrospective & $3 b$ & $111 / 70$ & $1,2,3,4,5,6$ & $* * * * * * *$ \\
\hline Ball, 2006 & USA & $2000-2005$ & Prospective & $3 b$ & $82 / 135$ & $1,3,5,6$ & $* * * * *$ \\
\hline Barocas, 2010 & USA & 2003-2008 & Prospective & $3 b$ & $1413 / 491$ & $1,3,7$ & $* * * * *$ \\
\hline Bolenz, 2010 & USA & 2003-2008 & Retrospective & $3 b$ & $262 / 161$ & $1,2,3,4,6$ & $* * * * * *$ \\
\hline Breyer, 2010 & USA & 2002-2008 & Prospective & $3 b$ & $293 / 695$ & $1,3,4,5,6,7$ & $* * * * * * *$ \\
\hline Carlsson, 2010 & Sweden & 2002-2007 & Prospective & $3 \mathrm{~b}$ & $1253 / 485$ & $1,3,4,5$ & ***** \\
\hline Chan, 2008 & USA & 2003-2006 & Retrospective & $3 \mathrm{~b}$ & $660 / 340$ & $1,3,5,6$ & ***** \\
\hline Chino, 2009 & USA & 2003-2007 & Retrospective & $3 b$ & $368 / 536$ & $1,3,5,6$ & ***** \\
\hline Choi, 2012 & Korea & 2007-2011 & Retrospective & $3 b$ & $354 / 247$ & $1,3,5$ & $* * * *$ \\
\hline Choo, 2013 & Korea & 2003-2010 & Prospective & $3 b$ & $77 / 176$ & $1,2,3,4,5,6,7$ & $* * * * * * *$ \\
\hline Chung, 2012 & Taiwan & 2006-2009 & Retrospective & 4 & $274 / 1773$ & 1,7 & $* * * *$ \\
\hline \begin{tabular}{|l|} 
D'Alonzo, 2009 \\
\end{tabular} & USA & 2003-2006 & Retrospective & $3 \mathrm{~b}$ & $256 / 280$ & $1,2,3,4,7$ & $* * * * * *$ \\
\hline Di Pierro, 2011 & Switzerland & 2007-2009 & Prospective & $3 b$ & $75 / 75$ & $1,3,5,6,7$ & $* * * * *$ \\
\hline Doumerc, 2010 & France & 2006-2008 & Prospective & $3 \mathrm{~b}$ & $212 / 502$ & $1,3,4,5,6,7$ & $* * * * * * *$ \\
\hline Drouin, 2009 & France & 2000-2004 & Retrospective & $3 \mathrm{~b}$ & $71 / 83$ & $1,2,3,5,6,7$ & $* * * * * * *$ \\
\hline Farnham, 2006 & USA & 2003-2004 & Prospective & $3 b$ & $176 / 103$ & $1,3,4,6$ & $* * * * *$ \\
\hline Ficarra, 2009 & Italy & 2006-2007 & Prospective & $3 b$ & $103 / 105$ & $1,2,3,4,5,6,7$ & $* * * * * * *$ \\
\hline Fracalanza, 2008 & Italy & 2006 & Prospective & $3 b$ & $35 / 26$ & $1,2,3,4,6$ & ****** \\
\hline Forehner, 2013 & Germany & 2007-2011 & Prospective & $3 b$ & $252 / 1925$ & $1,3,6,7$ & $* * * * *$ \\
\hline Hong, 2010 & Korea & 2007 & Retrospective & 4 & $26 / 25$ & $1,2,7$ & $* * * *$ \\
\hline \begin{tabular}{|l|} 
Park, 2014 \\
\end{tabular} & Korea & 2007-2012 & Retrospective & $3 b$ & $730 / 277$ & $1,2,3,4,5,6,7$ & $* * * * * * *$ \\
\hline Busch, 2015 & Germany & NA & Prospective & $3 \mathrm{~b}$ & $194 / 194$ & $1,3,5,6$ & $* * * * *$ \\
\hline Kim, 2011 & Korea & $2007-2010$ & Prospective & $3 b$ & $528 / 235$ & $1,2,3,5,6,7$ & ****** \\
\hline Kordan, 2010 & USA & 2003-2006 & Prospective & $3 b$ & $830 / 414$ & $1,2,3,5,6$ & $* * * * *$ \\
\hline Krambeck, 2008 & USA & 2002-2005 & Prospective & $3 b$ & $294 / 588$ & $1,2,3,5,6,7$ & $* * * * * * *$ \\
\hline Laurila, 2009 & USA & 2006 & Retrospective & $3 b$ & $94 / 98$ & $1,3,5,6$ & $* * * * *$ \\
\hline Lo, 2010 & HongKong & 2006-2007 & Retrospective & $3 b$ & $20 / 20$ & $1,3,5,6,7$ & $* * * * *$ \\
\hline Magheli, 2011 & USA & 2000-2008 & Prospective & $3 b$ & $522 / 522$ & $1,3,4,5,6,7$ & $* * * * *$ \\
\hline Malcolm, 2010 & USA & 2000-2008 & Retrospective & $3 b$ & $477 / 135$ & $1,3,5,6,7$ & ***** \\
\hline Menon, 2002 & France & 2001 & Prospective & $3 b$ & $30 / 30$ & $1,3,4,5,6,7$ & $* * * * * * *$ \\
\hline Miller, 2007 & USA & 2002-2006 & Prospective & 4 & $42 / 120$ & 1 & $* * * *$ \\
\hline Minniti, 2011 & Italy & 2007-2008 & Prospective & $3 b$ & $22 / 93$ & $1,2,3,5$ & $* * * *$ \\
\hline Nelson, 2007 & USA & 2003-2006 & Prospective & $3 b$ & $629 / 374$ & $1,3,6$ & ***** \\
\hline OU, 2009 & Taiwan & 2004-2007 & Retrospective & $3 b$ & $30 / 30$ & $1,2,3,4,5,6$ & $* * * * * * *$ \\
\hline Pilecki, 2014 & USA & 2011 & Retrospective & 4 & $4374 / 1097$ & 1,2 & $* * * *$ \\
\hline Rocco, 2009 & Italy & 2004-2007 & Prospective & $3 b$ & $120 / 240$ & $1,3,5,6,7$ & ****** \\
\hline Ryu, 2013 & Korea & 2007-2012 & Prospective & 4 & $524 / 341$ & $1,2,3,4$ & $* * * * *$ \\
\hline Schroeck, 2008 & USA & 2003-2007 & Retrospective & $3 b$ & $362 / 435$ & $1,2,3,4,5,6,7$ & $* * * * * * *$ \\
\hline Shapiro, 2014 & USA & $2000-2010$ & Retrospective & $3 b$ & $108 / 229$ & $1,3,5,6,7$ & $* * * * *$ \\
\hline Silberstein, 2012 & USA & 2010 & Retrospective & 4 & $126 / 126$ & $1,3,5,6$ & $* * * * *$ \\
\hline Smith, 2007 & USA & 2002-2006 & Retrospective & $3 b$ & $200 / 200$ & $1,2,3,4,5,6$ & $* * * * * * *$ \\
\hline Son, 2013 & Korea & 2006-2009 & Retrospective & $3 b$ & $146 / 112$ & $1,2,3,4,6,7$ & $* * * * * *$ \\
\hline Stranne, 2010 & Sweden & 2002-2006 & Retrospective & $3 b$ & $946 / 465$ & $1,2,3,4,5,6,7$ & $* * * * * * *$ \\
\hline Sugihara, 2014 & Japan & 2012-2013 & Retrospective & $3 b$ & $2126 / 7202$ & $1,2,5$ & $* * * *$ \\
\hline Tewari, 2003 & USA & 1999-2002 & Prospective & $3 b$ & $200 / 100$ & $1,2,3,4,5,6,7$ & $* * * * * * *$ \\
\hline Truesdale, 2010 & USA & 2005-2009 & Retrospective & $3 b$ & $99 / 217$ & $1,2,3,5,6$ & $* * * * * *$ \\
\hline Vora, 2013 & USA & 1997-2010 & Retrospective & $3 b$ & $140 / 95$ & $1,3,5,6,7$ & $* * * * *$ \\
\hline
\end{tabular}




\begin{tabular}{|c|c|c|c|c|c|c|c|}
\hline White, 2009 & USA & $2005-2008$ & Retrospective & $3 \mathrm{~b}$ & $50 / 50$ & $1,3,5,6$ & $* * * * *$ \\
\hline Williams, 2010 & USA & $2005-2008$ & Retrospective & 4 & $604 / 346$ & $1,3,5,6$ & $* * * * *$ \\
\hline Wood, 2007 & USA & $2003-2005$ & Prospective & 4 & $165 / 152$ & $1,3,7$ & $* * * * *$ \\
\hline Yi, 2010 & Korea & 2006-2009 & \begin{tabular}{|l|} 
Retrospective \\
\end{tabular} & $3 b$ & $153 / 641$ & $1,2,3,6,7$ & $* * * * *$ \\
\hline Rush, 2015 & Canada & 2009-2012 & Retrospective & $3 b$ & $331 / 643$ & $1,2,3,4,7$ & $* * * * *$ \\
\hline Ong, 2015 & Australian & 2009-2012 & Prospective & $3 b$ & $885 / 1117$ & $1,3,5,6,7$ & $* * * * * *$ \\
\hline Porcaro, 2015 & Italy & 2013 & Retrospective & 4 & $108 / 43$ & $1,2,3,4,5,6,7$ & $* * * * * * *$ \\
\hline O'Neil, 2015 & USA & $2011-2012$ & Prospective & $3 \mathrm{~b}$ & 933/1505 & $1,3,6,7$ & $* * * *$ \\
\hline Niklas, 2015 & Germany & $2003-2010$ & Retrospective & $3 b$ & $932 / 499$ & $1,2,3,4,5,6,7$ & $* * * * * * *$ \\
\hline Haglind, 2015 & Sweden & 2008-2011 & Prospective & $3 b$ & $1847 / 778$ & $1,2,3,5,6$ & $* * * * * *$ \\
\hline Gagnon, 2014 & Canada & NA & Retrospective & $3 \mathrm{~b}$ & $200 / 200$ & $1,2,3,4,5,6,7$ & $* * * * * *$ \\
\hline Davison, 2014 & Canada & $2007-2009$ & Prospective & $3 \mathrm{~b}$ & $78 / 73$ & $1,3,5$ & $* * * * *$ \\
\hline Akand, 2015 & Turkey & 1999-2012 & Retrospective & 4 & $79 / 50$ & $1,2,3,4,5,7$ & $* * * * * *$ \\
\hline Korets, 2014 & USA & $2007-2012$ & Retrospective & $3 \mathrm{~b}$ & $12746 / 3398$ & $1,2,7$ & $* * * * *$ \\
\hline Wallerstedt, 2015 & Sweden & NA & Prospective & $3 b$ & $1847 / 778$ & $1,2,3,5,6,7$ & $* * * * * *$ \\
\hline $\mathrm{Hu}, 2015$ & USA & 2004-2009 & Retrospective & $3 b$ & $5524 / 7878$ & $1,2,3,5,6,7$ & $* * * * * *$ \\
\hline Davis, 2014 & USA & 2004-2010 & Prospective & $3 b$ & $27348 / 13840$ & 1,7 & $* * * *$ \\
\hline Rithch, 2014 & USA & 2003-2009 & Retrospective & $3 \mathrm{~b}$ & $742 / 237$ & $1,2,3,5,6,7$ & $* * * * * *$ \\
\hline Gandaglia, 2014 & USA & 2008-2009 & Retrospective & $3 b$ & $3476 / 2439$ & $1,3,5,6,7$ & $* * * * *$ \\
\hline Koo, 2014 & Korea & $1992-2008$ & Retrospective & $3 \mathrm{~b}$ & $175 / 175$ & $1,3,5,6,7$ & $* * * * * *$ \\
\hline Busch, 2014 & Germany & NA & Retrospective & $3 \mathrm{~b}$ & $110 / 110$ & $1,2,3,4,5,6,7$ & $* * * * * * *$ \\
\hline Alemozaffar, 2015 & USA & $2000-2010$ & Prospective & $3 \mathrm{~b}$ & $282 / 621$ & $1,2,3,4,5,6,7$ & $* * * * * * *$ \\
\hline Harty, 2013 & USA & $2000-2010$ & Prospective & $3 b$ & $152 / 153$ & $1,3,4,5,6,7$ & $* * * * * * *$ \\
\hline Silberstein, 2013 & USA & $2007-2010$ & Retrospective & $3 \mathrm{~b}$ & $493 / 961$ & $1,3,5,7$ & $* * * * *$ \\
\hline Ludovico, 2013 & Italy & 2004-2008 & Retrospective & $3 \mathrm{~b}$ & $82 / 48$ & $1,3,5,6,7$ & $* * * * * *$ \\
\hline Musch, 2013 & Germany & $2009-2010$ & \begin{tabular}{|l|} 
Retrospective \\
\end{tabular} & $3 b$ & $105 / 105$ & $1,2,3,4,5,6,7$ & $* * * * * *$ \\
\hline Hall, 2014 & Australia & $2007-2009$ & Retrospective & $3 b$ & $100 / 100$ & $1,3,6$ & $* * * * *$ \\
\hline Geraerts, 2013 & Belgium & 2009-2011 & Prospective & $3 b$ & $64 / 116$ & $1,2,7$ & $* * * * *$ \\
\hline Drouin, 2014 & France & $2007-2010$ & Prospective & $3 b$ & $73 / 44$ & $1,3,5,6,7$ & $* * * * * *$ \\
\hline Pierorazio, 2013 & USA & $2002-2011$ & Retrospective & $3 \mathrm{~b}$ & $105 / 743$ & $1,2,3,4,5,6,7$ & $* * * * * * *$ \\
\hline
\end{tabular}

$\mathrm{RARP}=$ robot-assisted radical prostatectomy; $\mathrm{RRP}=$ retropubic radical prostatectomy; $\mathrm{NA}=$ data not available; $\mathrm{LOE}=$ level of evidence.

*:Matching/comparable variable: $1=$ age, $2=\mathrm{BMI}, 3=\mathrm{PSA}, 4=$ prostate size, $5=$ clinical stage, $6=$ biospy Gleason score, $7=$ follow up

$\Delta:$ based on Newcastle-Ottawa Scale.

surgery by expert indicate better ergonomics and quicker learning curve, but its shortage is high cost of the robotic surgery system.

In recent years, many experts have reported on comparative study of RARP and open RRP. And some meta-analysis were performed to evaluate the advantages and disadvantages of two approaches, including perioperative outcomes, oncologic outcomes [5]. Their early experience showed that the outcomes of this approach with fewer overall complications, quicker convalescence, and lower EBL and transfusion [5-7]. However, the outcomes of RARP compared with RRP have not been fully evaluated, and no conclusive results are available. Therefore, a systematic review and metaanalysis of the included published studies was performed to compare RARP with RRP.

\section{RESULTS}

\section{Characteristics of eligible studies}

According to search strategy, the included 78 studies[4, 8-85] assessing RARP $v s$. RRP met the inclusion criteria and were applied to perform this meta-analysis (Figure 1). Those studies include forty-three retrospective and thirty-five prospective studies and were listed in Table 1.

Quality of the studies and level of evidence (Table 1) In this meat-analysis, the Newcastle-Ottawa Scale quality assessment method of the observational studies [86], and the US Preventive Services Task Force grading system [87] were applied to evaluate the quality of included studies. Twenty studies scored seven stars and 
Table 2: Overall analysis of demographic and clinical characteristics compared RARP with RRP

\begin{tabular}{|c|c|c|c|c|c|c|c|c|}
\hline \multirow{2}{*}{ Outcomes of interest } & \multirow{2}{*}{$\begin{array}{l}\text { No. of } \\
\text { studies }\end{array}$} & \multirow{2}{*}{$\begin{array}{l}\text { No. of patients } \\
\text { RARP/RRP }\end{array}$} & \multirow{2}{*}{ OR/WMD(95\% CI ) } & \multirow{2}{*}{$p$-value } & \multicolumn{4}{|c|}{ Study heterogeneity } \\
\hline & & & & & $\mathrm{Chi}^{2}$ & df & $I^{2}$ & $p$-value \\
\hline Age(year) & 33 & $41866 / 227181$ & $-1.00[-1.56,-0.44]$ & $<0.001$ & 1260.51 & 32 & $97 \%$ & $<0.001$ \\
\hline $\operatorname{BMI}\left(\mathrm{kg} / \mathrm{m}^{2}\right)$ & 17 & $9365 / 4690$ & $-0.10[-0.39,0.20]$ & 0.52 & 87.93 & 16 & $82 \%$ & $<0.001$ \\
\hline Pre-PSA(ng/ml) & 23 & $6161 / 5250$ & $-0.93[-1.47,-0.40]$ & $<0.001$ & 234.69 & 22 & $91 \%$ & $<0.001$ \\
\hline Prostate volume $(\mathrm{ml})$ & 12 & $3995 / 3288$ & $2.35[-0.92,5.61]$ & 0.16 & 136.49 & 11 & $92 \%$ & $<0.001$ \\
\hline
\end{tabular}

$\mathrm{RARP}=$ robot-assisted radical prostatectomy; $\mathrm{RRP}=$ retropubic radical prostatectomy; $\mathrm{OR}=$ odds ratio; $\mathrm{WMD}=$ weighted mean difference; $\mathrm{CI}=$ confidence interval; $\mathrm{BMI}=$ body mass index.

were evaluated as the high quality studies. Additionally, The clinical variables of RARP and RRP were extracted independently from included literatures (Table 1).

\section{Description of included studies and patients Demographics (Table 2)}

Patients underwent RARP are younger $(\mathrm{WMD}=$ -1.00 years; $95 \% \mathrm{CI}:-1.56$ to $-0.44 ; P<0.001$ ) (Figure $\mathrm{S} 1)$, and have the lower level of pre-PSA (OR = -0.93; 95\% CI: -1.47 to $-0.40 ; P<0.001$ ) (Figure $\mathrm{S} 2$ ). But there is no significant difference on $\mathrm{BMI}(\mathrm{OR}=-0.10 ; 95 \% \mathrm{CI}$ : -0.39 to $0.20 ; P=0.20)$ (Figure $\mathrm{S} 3$ ), and prostate volume (WMD $=2.35 \mathrm{ml} ; 95 \% \mathrm{CI}:-0.92$ to $5.61 ; P=0.16$ ) (Figure S4) between the RARP and RRP group. (Table 2).

\section{Outcomes of perioperative variables (Table 3)}

\section{Operating time and estimated blood loss (EBL)}

With respect to perioperative variables, pooling data of 18 studies [21, 23, 24, 26, 29, 32, 34, 40, 54, 59, 60,
$64,70,75,78,81,84,85]$ involving 54261 participants indicated that RARP has longer operative time than RRP (WMD: 39.85 minutes; 95\% CI: 20.95 to 58.75; $P$ $<0.001$ ) (Figure 2). Pooling data of 13 studies [10, 21, $23,29,30,34,40,60,70,75,78,84,85]$ results showed that RARP has less intraoperative blood loss (WMD $=-507.67 \mathrm{ml} ; 95 \% \mathrm{CI}:-633.21$ to $-382.12 ; P<0.001)$ (Figure 3).

\section{Transfusion rate and postoperative recovery}

Pooled data from the 26 studies $[9,10,14,21,23$, $24,26,29,30,34,35,40,44-46,54,59,64,72,73,78,80$, $82,84]$ reported transfusion rate between RARP and RRP, and the results showed that RARP was associated with lower transfusion rate $(\mathrm{OR}=0.13 ; 95 \% \mathrm{CI}$ : 0.08 to $0.21 ; P$ $<0.001$ ) than RRP (Figure 4). Pooling data of 5 studies reported on the time to remove catheter, the forest plot showed that RARP had shorter time to remove catheter than RRP group (WMD $=-3.04 ; 95 \% \mathrm{CI}:-4.59$ to -1.49 ; $P<0.001$ ) (Figure S5). And pooling date of 11 studies $[10,23,24,34,53,54,64,75,78]$ reported on length of hospital stay (LOS), the forest plot showed that RARP had a shorter LOS than RRP (WMD $=-1.62 ; 95 \% \mathrm{CI}$ : -2.42 to $-0.82 ; P<0.001$ ) (Figure 5).

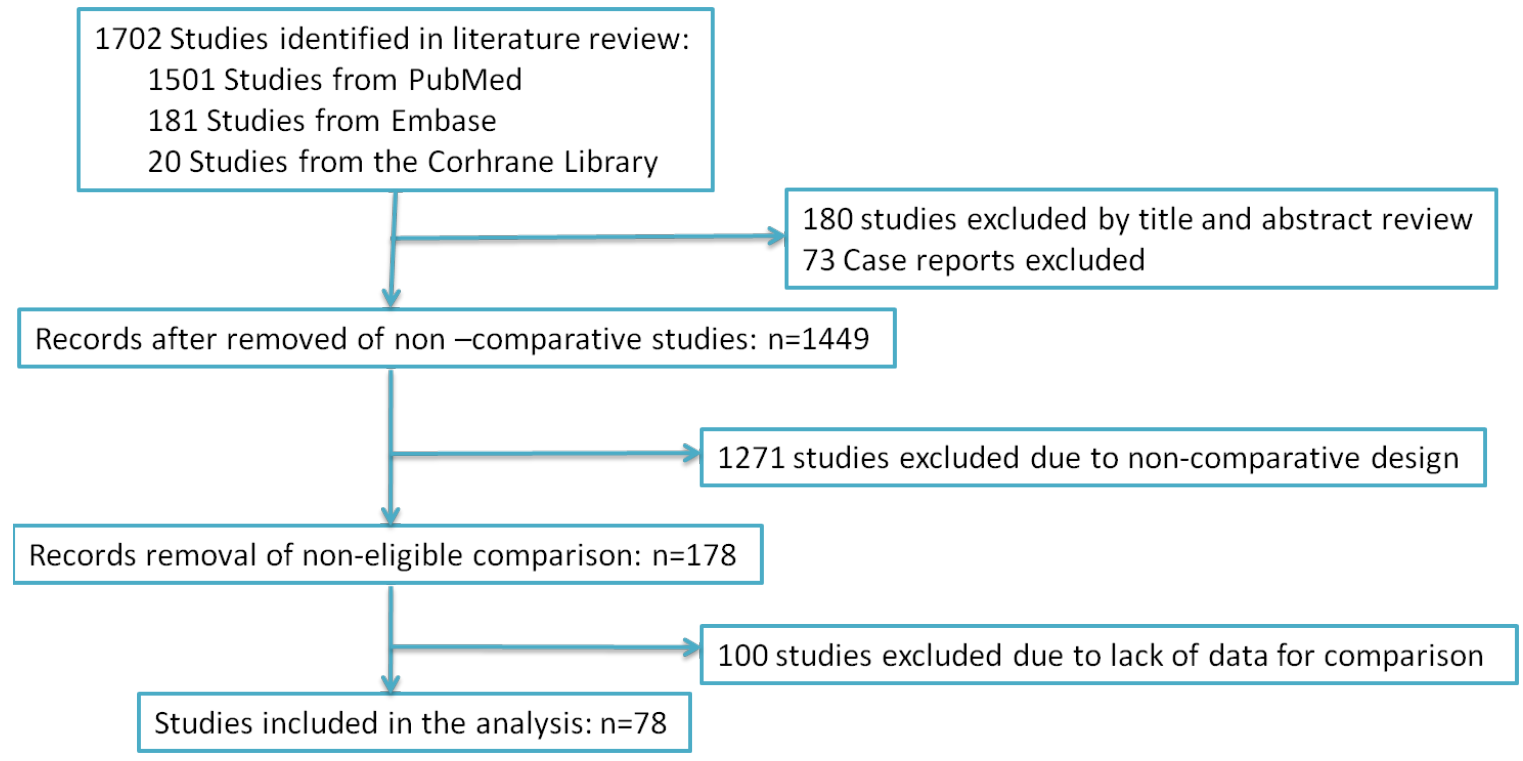

Figure 1: PRISMA diagram. The search strategy and number of studies identified for inclusion in this meta-analysis. 
Table 3: Overall analysis of perioperative outcomes comparing RARP with RRP

\begin{tabular}{|c|c|c|c|c|c|c|c|c|}
\hline \multirow{2}{*}{ Outcome of interest } & \multirow{2}{*}{$\begin{array}{l}\text { No. of } \\
\text { studies }\end{array}$} & \multirow{2}{*}{$\begin{array}{l}\text { No.of } \\
\text { patients } \\
\text { RARP/RRP }\end{array}$} & \multirow{2}{*}{ OR/WMD $(95 \% \mathrm{CI})^{\dagger}$} & \multirow{2}{*}{$p$-value } & \multicolumn{4}{|c|}{ Study heterogeneity } \\
\hline & & & & & $\mathrm{Chi}^{2}$ & df & $r^{2}$ & $p$-value \\
\hline Operation time, $\min$ & 18 & $36296 / 17965$ & $39.85[20.95,58.75]^{\dagger}$ & $<0.001$ & 2130.01 & 17 & $99 \%$ & $<0.001$ \\
\hline Estimated blood loss, ml & 13 & $3446 / 2791$ & $\begin{array}{l}-507.67[-633.21,- \\
382.12]^{\dagger}\end{array}$ & $<0.001$ & 390.34 & 12 & $97 \%$ & $<0.001$ \\
\hline Transfusion rate & 26 & $54847 / 32967$ & $0.13[0.08,0.21]$ & $<0.001$ & 693.85 & 25 & $96 \%$ & $<0.001$ \\
\hline Remove the catheter, day & 5 & $2135 / 1264$ & $-3.04[-4.59,-1.49]^{\dagger}$ & $<0.001$ & 260.52 & 4 & $98 \%$ & $<0.001$ \\
\hline Hospital stay, day & 11 & $32196 / 17106$ & $-1.62[-2.42,-0.82]^{\dagger}$ & $<0.001$ & 1517.19 & 10 & $99 \%$ & $<\mathbf{0 . 0 0 1}$ \\
\hline
\end{tabular}

$\mathrm{RARP}=$ robot-assisted radical prostatectomy; $\mathrm{RRP}=$ retropubic radical prostatectomy; $\mathrm{OR}=$ odds ratio; $\mathrm{WMD}=$ weighted mean difference; $\mathrm{CI}=$ confidence interval.

\begin{tabular}{|c|c|c|c|c|c|c|c|c|c|c|c|c|c|}
\hline \multirow[b]{2}{*}{ Study or Subgroup } & \multicolumn{3}{|c|}{ RARP } & \multicolumn{3}{|c|}{ RRP } & \multicolumn{2}{|r|}{ Mean Difference } & \multirow{2}{*}{\multicolumn{5}{|c|}{$\begin{array}{c}\text { Mean Difference } \\
\text { IV, Random, 95\% Cl }\end{array}$}} \\
\hline & Mean & SD & Total & Mean & SD & Total & Weight & IV, Random, $95 \% \mathrm{Cl}$ & & & & & \\
\hline Choo, 2013 & 220 & 62.12 & 77 & 151 & 44.02 & 176 & $5.6 \%$ & $69.00[53.68,84.32]$ & & & $\rightarrow$ & & \\
\hline D'Alonzo,2009 & 296 & 76 & 219 & 193 & 69 & 251 & $5.7 \%$ & $103.00[89.80,116.20]$ & & & & $\rightarrow$ & \\
\hline Davis 2014 & 264 & 102 & 27348 & 192 & 85.8 & 13840 & $5.8 \%$ & $72.00[70.13,73.87]$ & & & • & - & \\
\hline Di Pierro,2011 & 330 & 54 & 75 & 253 & 41 & 75 & $5.6 \%$ & $77.00[61.66,92.34]$ & & & & & \\
\hline Drouin,2009 & 199.6 & 36.6 & 71 & 208.5 & 76 & 83 & $5.5 \%$ & $-8.90[-27.33,9.53]$ & & & & & \\
\hline Fracalanza,2008 & 195.6 & 45 & 35 & 127.2 & 31.7 & 26 & $5.5 \%$ & $68.40[49.15,87.65]$ & & & & & \\
\hline Gagnon 2014 & 233.61 & 48.71 & 200 & 114.23 & 17.59 & 200 & $5.8 \%$ & $119.38[112.20,126.56]$ & & & & - & \\
\hline Hong, 2010 & 279.6 & 34.2 & 26 & 236.5 & 67.3 & 25 & $5.1 \%$ & $43.10[13.62,72.58]$ & & & & & \\
\hline Lo,2010 & 306 & 85 & 20 & 289 & 64 & 20 & $4.3 \%$ & $17.00[-29.63,63.63]$ & & & & & \\
\hline Niklas 2015 & 184.65 & 44.26 & 108 & 170.44 & 36.98 & 43 & $5.7 \%$ & $14.21[0.36,28.06]$ & & & & & \\
\hline OU,2009 & 205.2 & 102.6 & 30 & 213 & 37.2 & 30 & $4.7 \%$ & $-7.80[-46.85,31.25]$ & & & & & \\
\hline Pilecki,2014 & 212.3 & 73.7 & 4374 & 174 & 84.7 & 1097 & $5.8 \%$ & $38.30[32.83,43.77]$ & & & - & & \\
\hline Porcaro 2015 & 184.4 & 43.2 & 932 & 128 & 36 & 499 & $5.8 \%$ & $56.40[52.20,60.60]$ & & & - & & \\
\hline Ryu, 2013 & 146.4 & 47.4 & 524 & 170.8 & 61.3 & 341 & $5.8 \%$ & $-24.40[-32.07,-16.73]$ & & & & & \\
\hline Son, 2013 & 137.6 & 7.4 & 146 & 139.2 & 25 & 112 & $5.8 \%$ & $-1.60[-6.38,3.18]$ & & & & & \\
\hline Truesdale,2010 & 153.4 & 51.3 & 99 & 204 & 32.9 & 217 & $5.7 \%$ & $-50.60[-61.61,-39.59]$ & & & & & \\
\hline Wallerstedt 2015 & 175 & 168 & 1847 & 103 & 89 & 778 & $5.8 \%$ & $72.00[62.11,81.89]$ & & & & & \\
\hline Wood,2007 & 210 & 41.3 & 165 & 163 & 29 & 152 & $5.8 \%$ & $47.00[39.19,54.81]$ & & & - & & \\
\hline Total $(95 \% \mathrm{Cl})$ & & & 36296 & & & 17965 & $100.0 \%$ & $39.85[20.95,58.75]$ & & & & & \\
\hline \multicolumn{9}{|c|}{$\begin{array}{l}\text { Heterogeneity: } \operatorname{Tau}^{2}=1589.28 ; \mathrm{Chi}^{2}=2130.01, \mathrm{df}=17(\mathrm{P}<0.00001) ;\left.\right|^{2}=99 \% \\
\text { Test for overall effect: } Z=4.13(P<0.0001)\end{array}$} & -200 & $\begin{array}{l}-100 \\
\text { Favnurs RARP }\end{array}$ & ${ }^{0}$ Favnurs & $\begin{array}{r}100 \\
\text { RRP }\end{array}$ & 200 \\
\hline
\end{tabular}

Figure 2: Forest plot and meta-analysis of operating time between RARP and RRP. RARP = robot-assisted radical prostatectomy; RRP = retropubic radical prostatectomy.

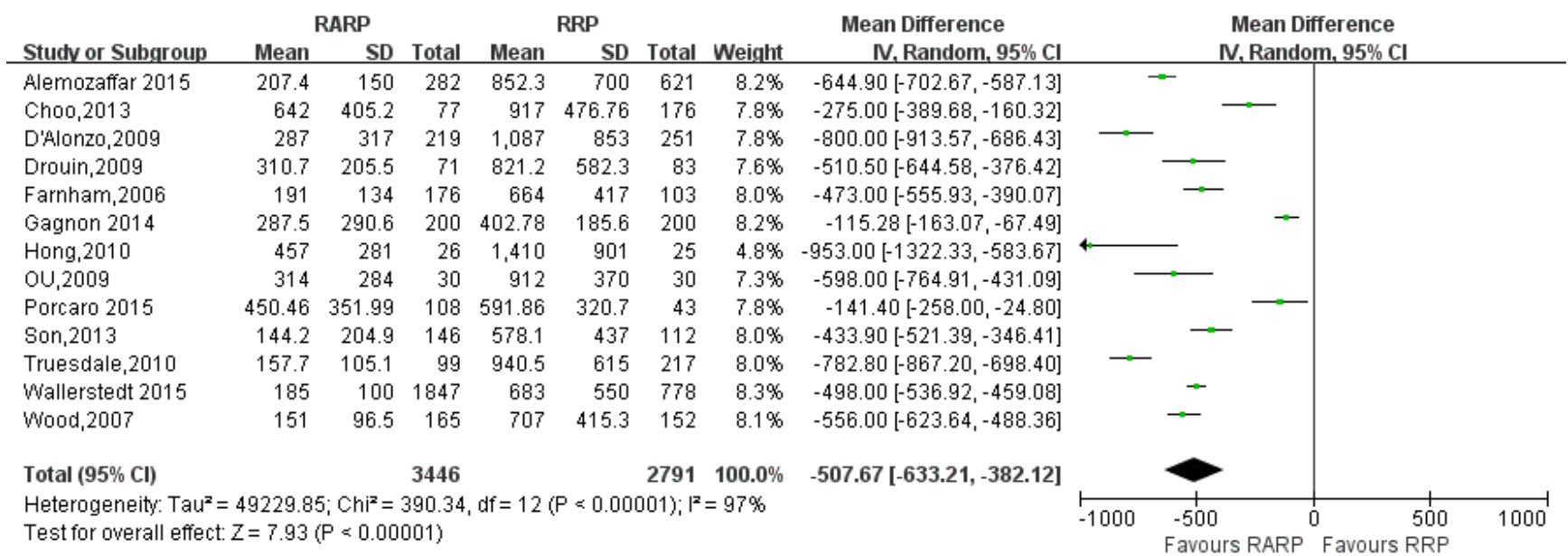

Figure 3: Forest plot and meta-analysis of estimated blood loss between RARP and RRP. RARP $=$ robot-assisted radical prostatectomy; RRP = retropubic radical prostatectomy. 


\section{Outcomes of oncological variables}

pathologic stage and pathologic Gleason score (Table 5)

14 studies $[9,20,27-29,32,46,48,66,70,73,76$,
$77,80]$ on $\leq \mathrm{pT} 2 \mathrm{a}, \mathrm{pT} 2 \mathrm{~b}, \geq \mathrm{pT} 2 \mathrm{c}, 48$ studies $[8-13,15,16$, $18,19,21,26-29,31,32,34,42-44,46-50,52,54,55,57$, $58,60,61,64-71,73,74,76-78,82,85]$ on pathologic Gleason score $(\leq 6 ; 7 ; \geq 8)$ were reported, respectively. The results showed a statistical differences more Gleason score

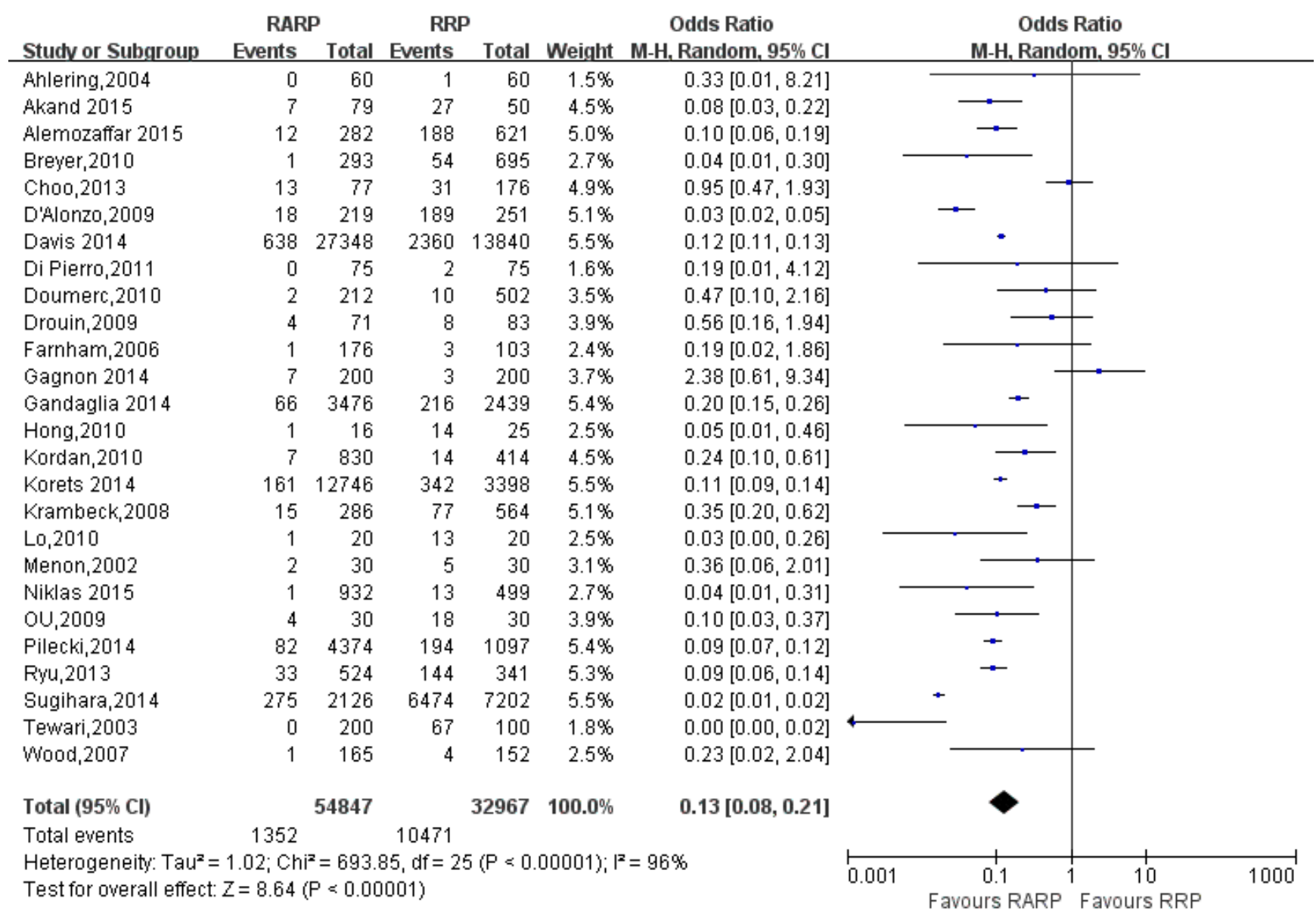

Figure 4: Forest plot and meta-analysis of transfusion rate between RARP and RRP. RARP = robot-assisted radical prostatectomy; RRP = retropubic radical prostatectomy.

\begin{tabular}{|c|c|c|c|c|c|c|c|c|c|c|c|c|}
\hline \multirow[b]{2}{*}{ Study or Subgroup } & \multicolumn{3}{|c|}{ RARP } & \multicolumn{3}{|c|}{ RRP } & \multicolumn{2}{|r|}{ Mean Difference } & \multirow{2}{*}{\multicolumn{4}{|c|}{$\begin{array}{l}\text { Mean Difference } \\
\text { IV. Random, } 95 \% \mathrm{Cl}\end{array}$}} \\
\hline & Mean & SD & Total & Mean & SD & Total & Weight & IV, Random, $95 \% \mathrm{Cl}$ & & & & \\
\hline Alemozaffar 2015 & 1.8 & 1 & 282 & 2.9 & 3 & 621 & $9.9 \%$ & $-1.10[-1.36,-0.84]$ & & $=$ & & \\
\hline D'Alonzo,2009 & 1.83 & 3.21 & 219 & 2.33 & 1.08 & 251 & $9.7 \%$ & $-0.50[-0.95,-0.05]$ & & $=$ & & \\
\hline Davis 2014 & 2.2 & 1.9 & 27348 & 3.7 & 2.7 & 13840 & $10.0 \%$ & $-1.50[-1.55,-1.45]$ & & ' & & \\
\hline Gagnon 2014 & 1.69 & 1.19 & 200 & 1.95 & 1.09 & 200 & $10.0 \%$ & $-0.26[-0.48,-0.04]$ & & & & \\
\hline Lo,2010 & 8 & 6 & 20 & 17 & 7 & 20 & $2.8 \%$ & $-9.00[-13.04,-4.96]$ & & & & \\
\hline Nelson,2007 & 1.17 & 1.03 & 629 & 1.23 & 1.09 & 374 & $10.0 \%$ & $-0.06[-0.20,0.08]$ & & & & \\
\hline Niklas 2015 & 8.7 & 2.2 & 932 & 15.2 & 3.6 & 499 & $9.8 \%$ & $-6.50[-6.85,-6.15]$ & & $*$ & & \\
\hline OU,2009 & 7.33 & 2.32 & 30 & 8.37 & 2.22 & 30 & $8.3 \%$ & $-1.04[-2.19,0.11]$ & & & & \\
\hline Ryu, 2013 & 7.9 & 5.1 & 524 & 10.1 & 3.2 & 341 & $9.6 \%$ & $-2.20[-2.75,-1.65]$ & & $\rightarrow$ & & \\
\hline Wallerstedt 2015 & 3.3 & 3 & 1847 & 4.1 & 4 & 778 & $9.9 \%$ & $-0.80[-1.11,-0.49]$ & & 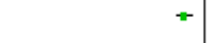 & & \\
\hline Wood,2007 & 1.2 & 0.84 & 165 & 1.3 & 0.95 & 152 & $10.0 \%$ & $-0.10[-0.30,0.10]$ & & & & \\
\hline Total $(95 \% \mathrm{Cl})$ & & & 32196 & & & 17106 & $100.0 \%$ & $-1.62[-2.42,-0.82]$ & & & & \\
\hline $\begin{array}{l}\text { Heterogeneity: } \mathrm{Tau}^{2} \\
\text { Test for overall effec }\end{array}$ & $\begin{array}{l}66 ; \mathrm{C} \\
=3.97\end{array}$ & & $\begin{array}{l}517.19, \mathrm{~d} \\
1.0001)\end{array}$ & & & & $=99 \%$ & & -10 & $\begin{array}{c}-5 \\
\text { Favours RARP }\end{array}$ & \begin{tabular}{|cc}
0 & 5 \\
Favours RRP
\end{tabular} & 10 \\
\hline
\end{tabular}

Figure 5: Forest plot and meta-analysis of the length of hospital stay between RARP and RRP. RARP = robot-assisted radical prostatectomy; RRP = retropubic radical prostatectomy. 
Table 4: Overall analysis of complications comparing RARP and RRP

\begin{tabular}{|c|c|c|c|c|c|c|c|c|}
\hline \multirow{2}{*}{ Outcome of interest } & \multirow{2}{*}{$\begin{array}{l}\text { No. of } \\
\text { studies }\end{array}$} & \multirow{2}{*}{$\begin{array}{l}\text { No.of patients } \\
\text { RARP/RRP }\end{array}$} & \multirow{2}{*}{ OR $(95 \% C I)$} & \multirow{2}{*}{$p$-value } & \multicolumn{4}{|c|}{ Study heterogeneity } \\
\hline & & & & & $\mathrm{Chi}^{2}$ & df & $I^{2}$ & $p$-value \\
\hline Overall complications & 25 & $43087 / 28834$ & $0.43[0.32,0.58]$ & $<0.001$ & 499.59 & 24 & $95 \%$ & $<0.001$ \\
\hline Rectal injury & 8 & $3888 / 8110$ & $0.16[0.07,0.39]$ & $<0.001$ & 5.22 & 7 & $0 \%$ & 0.63 \\
\hline Pulmonary embolism & 9 & $37575 / 24635$ & $0.47[0.37,0.59]$ & $<0.001$ & 5.04 & 8 & $0 \%$ & 0.75 \\
\hline Wound infections & 10 & $11161 / 10587$ & $0.23[0.11,0.46]$ & $<0.001$ & 31.49 & 9 & $71 \%$ & $<0.001$ \\
\hline Bladder neck contracture & 4 & $1993 / 2409$ & $0.21[0.08,0.60]$ & 0.003 & 8.39 & 3 & $64 \%$ & 0.04 \\
\hline UTI & 4 & $6586 / 2546$ & $0.75[0.37,1.54]$ & 0.44 & 15.35 & 3 & $80 \%$ & 0.002 \\
\hline Urinary retention & 3 & $2042 / 960$ & $0.63[0.47,0.84]$ & 0.002 & 2.44 & 2 & $18 \%$ & 0.29 \\
\hline Obturator nerve injury & 2 & $1453 / 585$ & $0.09[0.01,0.75]$ & 0.03 & 0.01 & 1 & $0 \%$ & 0.91 \\
\hline DVT & 7 & $7479 / 3072$ & $0.40[0.25,0.66]$ & $<0.001$ & 10.82 & 6 & $45 \%$ & 0.09 \\
\hline Urinary leakage & 8 & $30940 / 15631$ & $0.64[0.58,0.70]$ & $<0.001$ & 8.87 & 7 & $21 \%$ & 0.26 \\
\hline ileus & 8 & $3412 / 8501$ & $0.92[0.56,1.51]$ & 0.73 & 2.20 & 7 & $0 \%$ & 0.95 \\
\hline lymphocele & 9 & $45258 / 2639$ & $0.52[0.29,0.94]$ & 0.03 & 8.93 & 8 & $10 \%$ & 0.35 \\
\hline Urinary continence- $3 \mathrm{mo}$ & 9 & 997/941 & $1.54[0.92,2.58]$ & 0.10 & 22.06 & 8 & $64 \%$ & 0.005 \\
\hline Urinary continence- $12 \mathrm{mo}$ & 9 & $1565 / 2179$ & $1.03[0.84,1.27]$ & 0.75 & 17.41 & 8 & $54 \%$ & 0.03 \\
\hline Potent recovery-3mo & 5 & $1169 / 820$ & $3.19[1.19,8.56]$ & 0.02 & 51.94 & 4 & $92 \%$ & $<0.001$ \\
\hline Potent recovery-12mo & 7 & $1395 / 1574$ & $2.37[1.30,4.33]$ & 0.005 & 55.43 & 6 & $89 \%$ & $<0.001$ \\
\hline Readmission rate & 7 & $11632 / 7060$ & $0.83[0.74,0.94]$ & 0.002 & 36.82 & 6 & $84 \%$ & $<0.001$ \\
\hline
\end{tabular}

$\mathrm{RARP}=$ robot-assisted radical prostatectomy; $\mathrm{RRP}=$ retropubic radical prostatectomy; $\mathrm{OR}=$ odds ratio; $\mathrm{WMD}=\mathrm{weighted}$ mean difference; $\mathrm{CI}=$ confidence interval; UTI=urinary tract infection; DVT=deep venous thrombosis.

Table 5: Overall analysis of pathologic and oncological outcomes comparing RARP with RRP

\begin{tabular}{|c|c|c|c|c|c|c|c|c|}
\hline \multirow{2}{*}{ Outcome of interest } & \multirow{2}{*}{$\begin{array}{l}\text { No. of } \\
\text { studies }\end{array}$} & \multirow{2}{*}{\begin{tabular}{|l|} 
No.of \\
patients \\
RARP/RRP
\end{tabular}} & \multirow{2}{*}{ OR/WMD(95\%CI) } & \multirow{2}{*}{$p$-value } & \multicolumn{4}{|c|}{ Study heterogeneity } \\
\hline & & & & & $\mathrm{Chi}^{2}$ & df & $P^{2}$ & $p$-value \\
\hline \multicolumn{9}{|l|}{\begin{tabular}{|l} 
Pathologic T stage \\
\end{tabular}} \\
\hline$\leq \mathrm{pT} 2 \mathrm{a}$ & 13 & $2147 / 2174$ & $1.11[0.93,1.31]$ & 0.26 & 8.84 & 12 & $0 \%$ & 0.72 \\
\hline pT2b & 11 & $1959 / 2098$ & $1.11[0.93,1.33]$ & 0.25 & 13.91 & 10 & $28 \%$ & 0.18 \\
\hline$\geq \mathrm{pT} 2 \mathrm{c}$ & 14 & $2268 / 2485$ & $0.93[0.76,1.13]$ & 0.44 & 11.19 & 13 & $0 \%$ & 0.60 \\
\hline \multicolumn{9}{|c|}{\begin{tabular}{|l} 
Pathological Gleason score \\
\end{tabular}} \\
\hline$\leq 6$ & 48 & $15238 / 13412$ & $1.04[0.91,1.18]$ & 0.61 & 224.21 & 47 & $79 \%$ & $<0.001$ \\
\hline 7 & 48 & $15238 / 13412$ & $1.17[1.04,1.33]$ & 0.01 & 230.23 & 47 & $80 \%$ & $<0.001$ \\
\hline$\geq 8$ & 48 & $15238 / 13412$ & $0.68[0.60,0.78]$ & $<0.001$ & 101.358 & 47 & $54 \%$ & $<0.001$ \\
\hline PSM & 49 & $20804 / 23133$ & $0.88[0.78,1.00]$ & 0.04 & 198.74 & 48 & $76 \%$ & $<0.001$ \\
\hline PSM for T2 & 28 & $10086 / 9711$ & $0.77[0.63,0.95]$ & 0.01 & 82.23 & 27 & $67 \%$ & $<0.001$ \\
\hline PSM for T3 & 18 & $2011 / 2125$ & $1.46[1.27,1.67]$ & $<0.001$ & 18.66 & 17 & $9 \%$ & 0.35 \\
\hline Mean lymph node yield & 4 & $837 / 565$ & $2.85[-0.92,6.63]^{\dagger}$ & 0.14 & 115.32 & 3 & $97 \%$ & $<0.001$ \\
\hline Positive lymph node & 16 & $4162 / 6500$ & $0.45[0.31,0.65]$ & $<0.001$ & 32.02 & 15 & $53 \%$ & 0.006 \\
\hline BCR for free survival & 10 & $4342 / 4176$ & $1.33[1.01,1.76]$ & 0.04 & 39.04 & 9 & $77 \%$ & $<0.001$ \\
\hline
\end{tabular}

$\mathrm{RARP}=$ robot-assisted radical prostatectomy; $\mathrm{RRP}=$ retropubic radical prostatectomy; $\mathrm{OR}=$ odds ratio; $\mathrm{WMD}=$ weighted mean difference; $\mathrm{CI}=$ confidence interval; PSM=positive surgical margins; ${ }^{\dagger}$ value of WMD.

$=7(\mathrm{OR}: 1.17 ; 95 \% \mathrm{CI}: 1.04$ to $1.33 ; P=0.01$; Figure 6) performed RARP and more Gleason score $\geq 8$ (OR: 0.68; $95 \%$ CI: 0.60 to $0.78 ; P<0.001$; Figure 6 ) in RRP. However, there were no statistical differences with respect to Gleason score $\leq 6$ (OR: $1.04 ; 95 \% \mathrm{CI}: 0.91$ to $1.18 ; P=$ 0.61 ; Figure 6 ) and pathologic T stage in the two groups (Figure S6,7,8)(Table 5).
Positive surgical margins and lymph node yield(Table 5)

49 studies [9-12, 14-16, 18, 19, 21, 26-29, 31-34, $36,37,39,41-44,46-49,52,54,56-58,61,62,65,67-$ 69, 73, 76-78, 80-82, 84] evaluating RARP and RRP reported positive surgical margins(PSM) rates. The results showed a significant difference with higher PSM rates in RRP group (OR:0.88; 95\% CI: 0.78 to $1.00 ; P=$ 


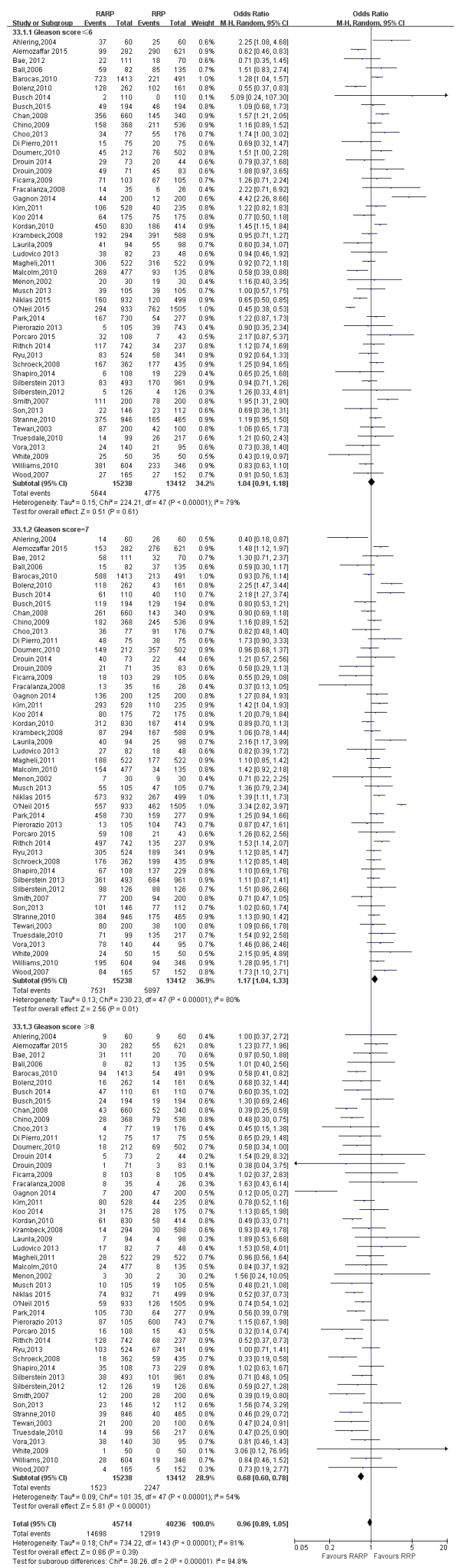

Figure 6: Forest plot and meta-analysis of pathological Gleason Score between RARP and RRP. RARP = robot-assisted radical prostatectomy; RRP = retropubic radical prostatectomy. 
Table 6: Sensitivity analysis of high quality studies comparing RARP with RRP

\begin{tabular}{|c|c|c|c|c|c|c|c|c|}
\hline \multirow{2}{*}{ Outcome of interest } & \multirow{2}{*}{$\begin{array}{l}\text { No. of } \\
\text { studies }\end{array}$} & \multirow{2}{*}{\begin{tabular}{|l|} 
No.of \\
patients \\
RARP/RRP
\end{tabular}} & \multirow{2}{*}{ OR/WMD $(95 \% \mathrm{CI})^{\dagger}$} & \multirow{2}{*}{$p$-value } & \multicolumn{4}{|c|}{ Study heterogeneity } \\
\hline & & & & & $\mathrm{Chi}^{2}$ & $\mathrm{df}$ & $I^{2}$ & $p$-value \\
\hline Operation time, $\min$ & 10 & $1523 / 1435$ & $44.43[8.01,80.84]^{\dagger}$ & 0.02 & 1166.56 & 9 & $99 \%$ & $<0.0001$ \\
\hline Estimated blood loss, ml & 8 & $1080 / 1102$ & $\begin{array}{l}-493.41[-672.09,- \\
314.74]^{\dagger}\end{array}$ & $<0.001$ & 217.36 & 7 & $97 \%$ & $<0.0001$ \\
\hline Transfusion rate & 18 & $16249 / 7209$ & $0.16[0.09,0.28]$ & $<0.001$ & 116.44 & 17 & $85 \%$ & $<0.0001$ \\
\hline Remove the catheter, day & 3 & $1173 / 735$ & $-1.78[-2.50,-1.06]^{\dagger}$ & $<0.001$ & 19.52 & 2 & $90 \%$ & $<0.0001$ \\
\hline Hospital stay, day & 6 & $1568 / 1117$ & $-0.75[-1.26,-0.24]^{\dagger}$ & 0.004 & 75.72 & 5 & $93 \%$ & $<0.0001$ \\
\hline Overall complications & 14 & $2782 / 2767$ & $0.50[0.27,0.92]$ & 0.03 & 158.13 & 13 & $92 \%$ & $<0.0001$ \\
\hline Urinary continence- $3 \mathrm{mo}$ & 7 & $945 / 818$ & $1.21[0.74,1.98]$ & 0.45 & 13.33 & 6 & $55 \%$ & 0.04 \\
\hline $\begin{array}{l}\text { Urinary continence- } \\
12 \mathrm{mo}\end{array}$ & 4 & $942 / 1409$ & $0.97[0.78,1.20]$ & 0.79 & 10.89 & 6 & $45 \%$ & 0.09 \\
\hline Potent recovery-3mo & 4 & $722 / 685$ & $4.50[1.91,10.62]$ & $<0.001$ & 17.64 & 3 & $83 \%$ & $<0.001$ \\
\hline Potent recovery- $12 \mathrm{mo}$ & 4 & $942 / 1409$ & $1.58[1.05,2.36]$ & 0.03 & 10.33 & 3 & $71 \%$ & 0.02 \\
\hline Readmission rate & 4 & $2850 / 3025$ & $0.53[0.23,1.21]$ & 0.13 & 24.10 & 3 & $88 \%$ & $<0.001$ \\
\hline \multicolumn{9}{|l|}{ Pathologic T stage } \\
\hline$\leq \mathrm{pT} 2 \mathrm{a}$ & 10 & $1725 / 1871$ & $1.02[0.83,1.26]$ & 0.83 & 7.02 & 9 & $0 \%$ & 0.63 \\
\hline pT2b & 9 & $1675 / 1821$ & $0.99[0.80,1.21]$ & 0.90 & 8.07 & 8 & $1 \%$ & 0.43 \\
\hline$\geq \mathrm{pT} 2 \mathrm{c}$ & 12 & $1979 / 2212$ & $0.98[0.79,1.21]$ & 0.84 & 9.48 & 11 & $0 \%$ & 0.58 \\
\hline \multicolumn{9}{|l|}{$\begin{array}{l}\begin{array}{l}\text { Pathological Gleason } \\
\text { score }\end{array} \\
\end{array}$} \\
\hline$\leq 6$ & 27 & $5847 / 6576$ & $0.99[0.87,1.13]$ & 0.88 & 45.37 & 26 & $43 \%$ & 0.01 \\
\hline 7 & 27 & $5847 / 6576$ & $1.14[1.02,1.28]$ & 0.02 & 46.80 & 26 & $44 \%$ & 0.007 \\
\hline$\geq 8$ & 27 & $5847 / 6576$ & $0.79[0.67,0.92]$ & 0.003 & 38.31 & 26 & $32 \%$ & 0.06 \\
\hline PSM & 39 & $13992 / 17806$ & $0.87[0.76,0.99]$ & 0.04 & 123.38 & 37 & $70 \%$ & $<0.001$ \\
\hline PSM for T2 & 16 & $6649 / 7986$ & $0.71[0.53,0.95]$ & 0.02 & 51.53 & 15 & $71 \%$ & $<0.001$ \\
\hline PSM for T3 & 12 & $1423 / 1713$ & $1.39[1.19,1.63]$ & $<0.001$ & 9.38 & 11 & $0 \%$ & 0.59 \\
\hline Mean lymph node yield & 2 & $375 / 275$ & $3.77[-5.87,13.41]^{\dagger}$ & 0.44 & 106.54 & 1 & $99 \%$ & $<0.001$ \\
\hline Positive lymph node & 10 & $2668 / 3684$ & $0.69[0.52,0.90]$ & 0.006 & 9.31 & 9 & $3 \%$ & 0.41 \\
\hline $\mathrm{BCR}$ for free survival & 5 & $1192 / 1797$ & $1.16[0.71,1.89]$ & 0.55 & 23.76 & 4 & $83 \%$ & $<0.001$ \\
\hline
\end{tabular}

$\mathrm{RARP}=$ robot-assisted radical prostatectomy; $\mathrm{RRP}=$ retropubic radical prostatectomy; $\mathrm{OR}=$ odds ratio; $\mathrm{WMD}=$ weighted mean difference; $\mathrm{CI}=$ confidence interval.

0.04)(Figure 7). PSM rates in pT3 cancers was higher in RARP group (OR:1.46; 95\% CI: 1.27 to $1.67 ; P<0.001$ ) (Figure 8). However, the results showed that PSM rates in pT2 cancers was lower in RARP (OR:0.77; 95\% CI: 0.63 to $0.95 ; P=0.01$ )(Figure 9 ). Four studies [20, 43, $60,73]$ comparing mean lymph node yield and the results showed that lymph node yield is higher in RARP (WMD: $1.61 ; 95 \%$ CI: 1.18 to $2.05 ; P<0.001$ )(Figure S9), and 16 studies $[20,26,33,34,39,49,58,61,64-68,73,84,85]$ reported on positive lymph node, There was a statistical differences decreased positive lymph node in RARP than RRP (OR:0.45; 95\% CI: 0.31 to $0.65 ; P<0.001$ )(Figure $10)$.

\section{Outcomes of complications(Table 4)}

Pooling data from 25 studies [9, 11, 17, 23, 24, $26,27,29,31,34,35,40,42,46,48,52-54,59,64$, $72,73,80,82,84]$ reported on overall complications, RARP had lower overall complications in the RARP than RRP(OR:0.43; 95\% CI: 0.32 to $0.58 ; P<0.001)$ (Figure 11). Next, a meticulous classification of overall complications showed that RRP had a higher incidence of rectal injury(OR:0.16; $95 \%$ CI: 0.07 to $0.39 ; P<0.001$ ) (Figure S10), pulmonary embolism(OR:0.47; 95\% CI: 0.37 to $0.59 ; P<0.001$ ) (Figure S11), wound infections (OR:0.23; 95\% CI: 0.11 to $0.46 ; P<0.001$ ) (Figure $\mathrm{S} 12$ ), bladder neck contracture(OR: $0.21 ; 95 \%$ CI: 0.08 to 0.60 ; $P=0.003$ ) (Figure S13), urinary retention(OR:0.63; 95\% CI: 0.47 to $0.84 ; P=0.002$ )(Figure $\mathrm{S} 14$ ), deep venous thrombosis(OR:0.40; 95\% CI: 0.25 to $0.66 ; P<0.001$ ) (Figure S15), urinary leakage(OR: $0.64 ; 95 \%$ CI: 0.58 to $0.70 ; P<0.001)$ (Figure S16), lymphocele (OR:0.52; 95\% CI: 0.29 to $0.94 ; P=0.03$ ) (Figure S17), and obturator nerve injury(OR:0.09; $95 \% \mathrm{CI}: 0.01$ to $0.75 ; P=0.03$ ) (Figure S18). There was no statistical differences between two groups in term of urinary tract infections(UTI) (OR:0.75; 95\% CI: 0.37 to $1.54 ; P=0.44$ )(Figure $\mathrm{S} 19$ ), ileus (OR:0.92; 95\% CI: 0.56 to $1.51 ; P=0.73$ ) (Figure S20). 
Urinary continence recovery and potent recovery(Table 4)

Pooling data of 9 studies $[9,21,26,50,62,70,81$, $83,84]$ reported on 3-mo and 12-mo urinary continence recovery between two groups. The forest plot showed that there were no statistical differences on the 3-mo and 12-mo urinary continence between two groups (3mo: OR:1.54; $95 \%$ CI: 0.92 to $2.58 ; P=0.10 ; 12 \mathrm{mo}$ : OR:1.03; $95 \% \mathrm{CI}: 0.84$ to $1.27 ; P=0.75$,respectively)(Figure 12 ,

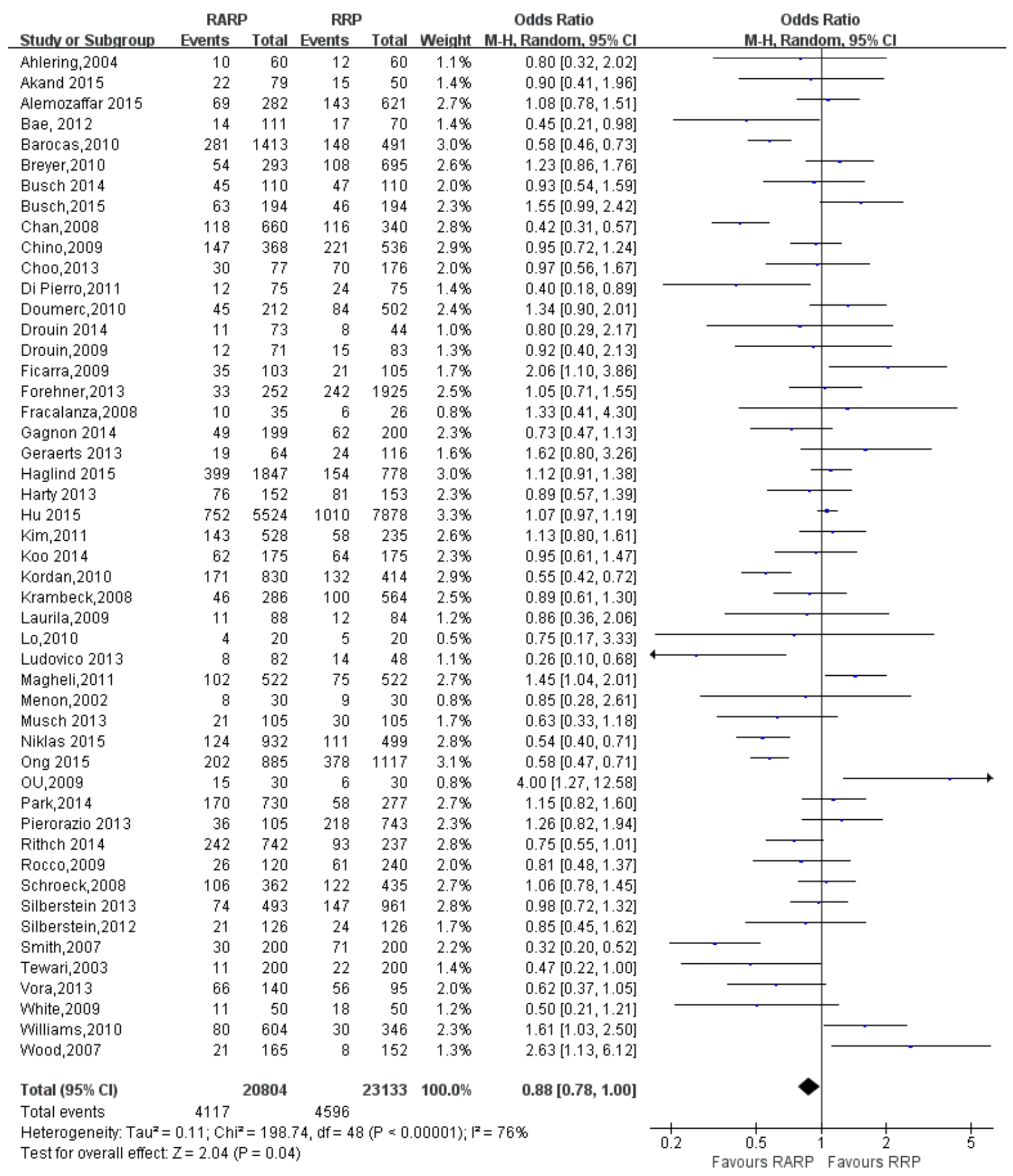

Figure 7: Forest plot and meta-analysis of PSM between RARP and RRP. RARP = robot-assisted radical prostatectomy; RRP $=$ retropubic radical prostatectomy. 


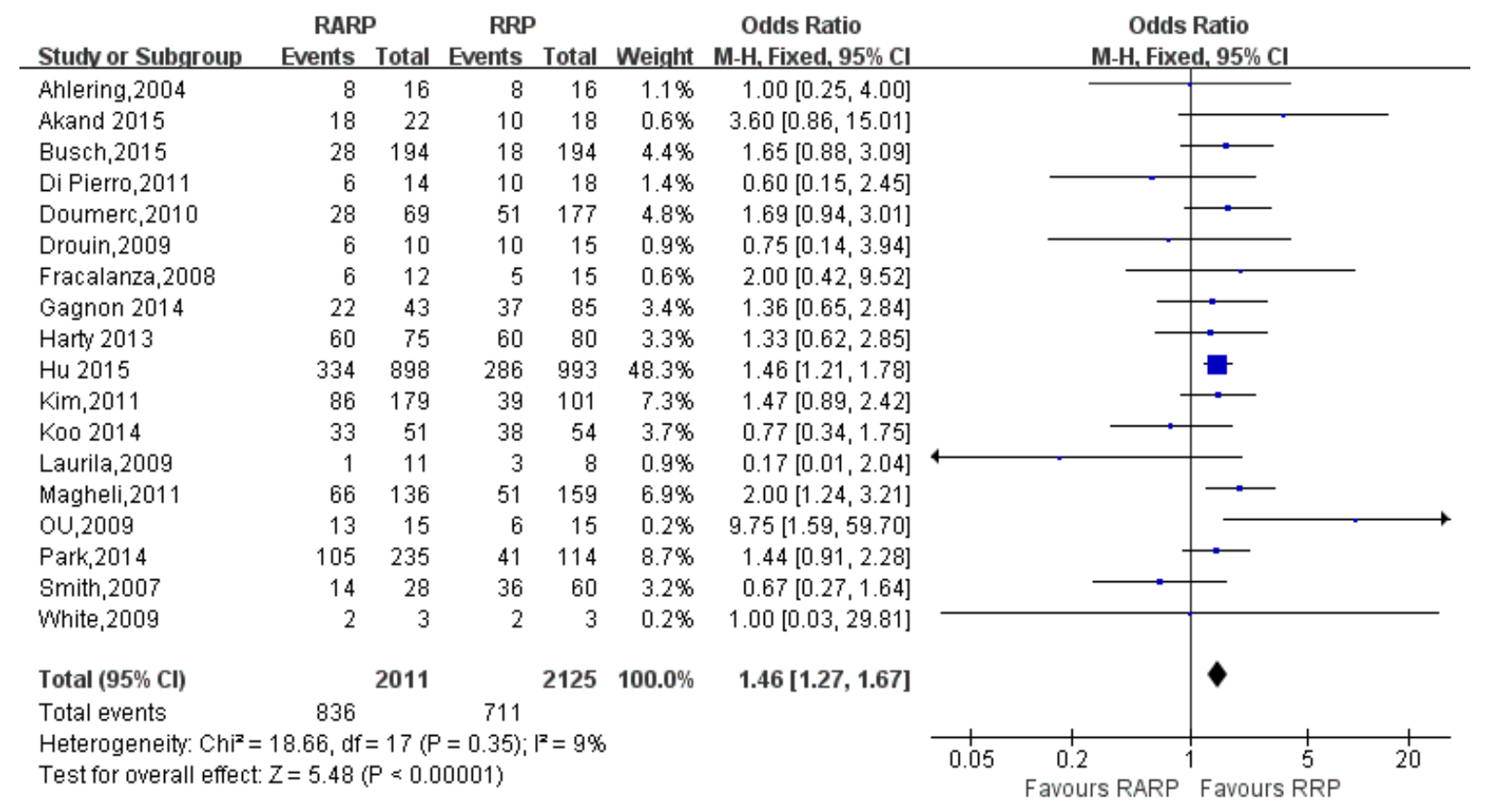

Figure 8: Forest plot and meta-analysis of PSM for pT3 between RARP and RRP. RARP= robot-assisted radical prostatectomy; $\mathrm{RRP}=$ retropubic radical prostatectomy.

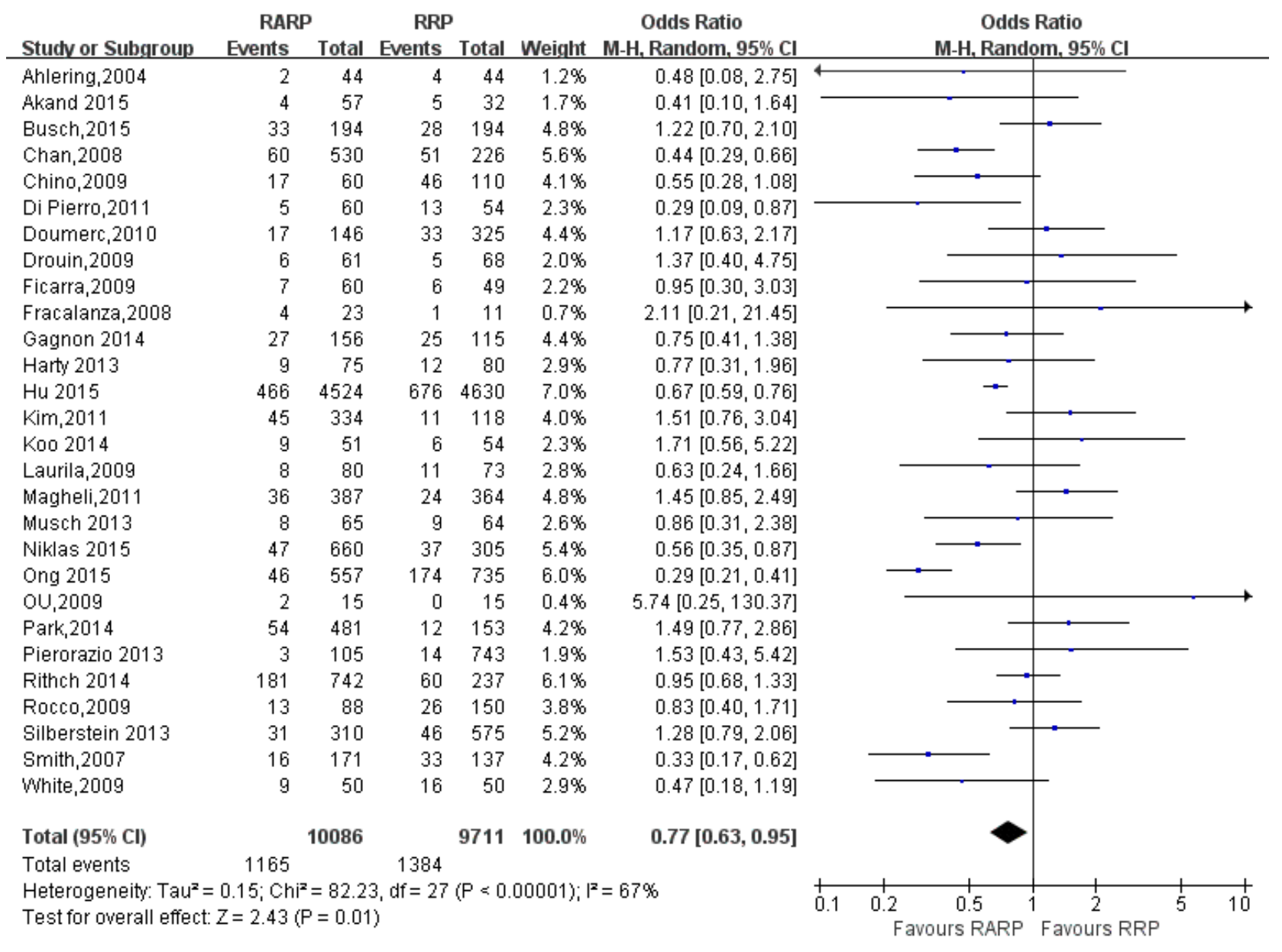

Figure 9: Forest plot and meta-analysis of PSM for pT2 between RARP and RRP. RARP= robot-assisted radical prostatectomy; $\mathrm{RRP}=$ retropubic radical prostatectomy. 
Figure S21). And the 3- and 12-mo potent recovery rate of RARP were better than RRP group, respectively (OR:3.19; 95\% CI: 1.19 to $8.56 ; P=0.02$; OR: $2.37 ; 95 \% \mathrm{CI}: 1.30$ to $4.33 ; P=0.005$,respectively)(Figure 13,14).

\section{Biochemical recurrence free survival and Readmission rate(Table 5)}

Pooling data from 10 studies[12, 16, 34, 49, 56, 61, $65-67,74]$ reported on biochemical recurrence(BCR) free survival, these results showed that RARP had a better BCR

\begin{tabular}{|c|c|c|c|c|c|c|c|c|c|c|}
\hline Study or Subgroup & $\begin{array}{l}\text { RARP } \\
\text { Events }\end{array}$ & Total & $\begin{array}{l}\text { RRP } \\
\text { Events }\end{array}$ & Total & Weight & $\begin{array}{c}\text { Odds Ratio } \\
\text { M-H, Random, } 95 \% \mathrm{Cl}\end{array}$ & & $\begin{array}{r}\text { Odds } \\
\text { M-H, Randc }\end{array}$ & $\begin{array}{l}\text { Ratio } \\
\text { lom, } 95 \% \mathrm{Cl}\end{array}$ & \\
\hline Choi,2012 & 7 & 354 & 14 & 247 & $7.6 \%$ & $0.34[0.13,0.84]$ & & $\longrightarrow$ & & \\
\hline Di Pierro,2011 & 9 & 75 & 12 & 75 & $7.6 \%$ & $0.72[0.28,1.82]$ & & & 1 & \\
\hline Forehner,2013 & 4 & 252 & 176 & 1925 & $7.1 \%$ & $0.16[0.06,0.44]$ & & & & \\
\hline Gagnon 2014 & 2 & 39 & 18 & 165 & $4.3 \%$ & $0.44[0.10,1.99]$ & & & & \\
\hline Harty 2013 & 5 & 153 & 4 & 152 & $5.1 \%$ & $1.25[0.33,4.75]$ & & & & \\
\hline Magheli,2011 & 0 & 522 & 5 & 522 & $1.5 \%$ & $0.09[0.00,1.63]$ & & & . & \\
\hline OU,2009 & 2 & 30 & 3 & 30 & $3.1 \%$ & $0.64[0.10,4.15]$ & & & & \\
\hline Pierorazio 2013 & 4 & 105 & 80 & 743 & $6.9 \%$ & $0.33[0.12,0.92]$ & & & & \\
\hline Rithch 2014 & 18 & 720 & 26 & 232 & $10.3 \%$ & $0.20[0.11,0.38]$ & & & & \\
\hline Ryu, 2013 & 13 & 524 & 15 & 341 & $9.0 \%$ & $0.55[0.26,1.18]$ & & & & \\
\hline Schroeck,2008 & 1 & 362 & 8 & 435 & $2.6 \%$ & $0.15[0.02,1.19]$ & & & & \\
\hline Shapiro,2014 & 8 & 108 & 17 & 229 & $8.0 \%$ & $1.00[0.42,2.39]$ & & & & \\
\hline Silberstein 2013 & 41 & 493 & 97 & 961 & $12.5 \%$ & $0.81[0.55,1.18]$ & & & & \\
\hline Silberstein, 2012 & 16 & 126 & 22 & 126 & $9.6 \%$ & $0.69[0.34,1.38]$ & & $\rightarrow$ & & \\
\hline Tewari,2003 & 1 & 200 & 2 & 100 & $2.0 \%$ & $0.25[0.02,2.75]$ & & & & \\
\hline Truesdale,2010 & 1 & 99 & 19 & 217 & $2.7 \%$ & $0.11[0.01,0.81]$ & & & & \\
\hline Total $(95 \% \mathrm{Cl})$ & & 4162 & & 6500 & $100.0 \%$ & $0.45[0.31,0.65]$ & & & & \\
\hline \multirow{2}{*}{\multicolumn{7}{|c|}{$\begin{array}{l}\text { Heterogeneity: } \text { Tau }^{2}=0.25 ; \mathrm{Chi}^{2}=32.02, \mathrm{df}=15(\mathrm{P}=0.006) ;\left.\right|^{2}=53 \% \\
\text { Test for overall effect: } Z=4.25(\mathrm{P}=0.0001)\end{array}$}} & & & & \\
\hline & & & & & & & 0.005 & $\begin{array}{c}0.1 \\
\text { Favours RARP }\end{array}$ & $\begin{array}{l}10 \\
\text { Favours RF }\end{array}$ & 200 \\
\hline
\end{tabular}

Figure 10: Forest plot and meta-analysis of positive lymph node between RARP and RRP. RARP $=$ robot-assisted radical prostatectomy; RRP = retropubic radical prostatectomy.

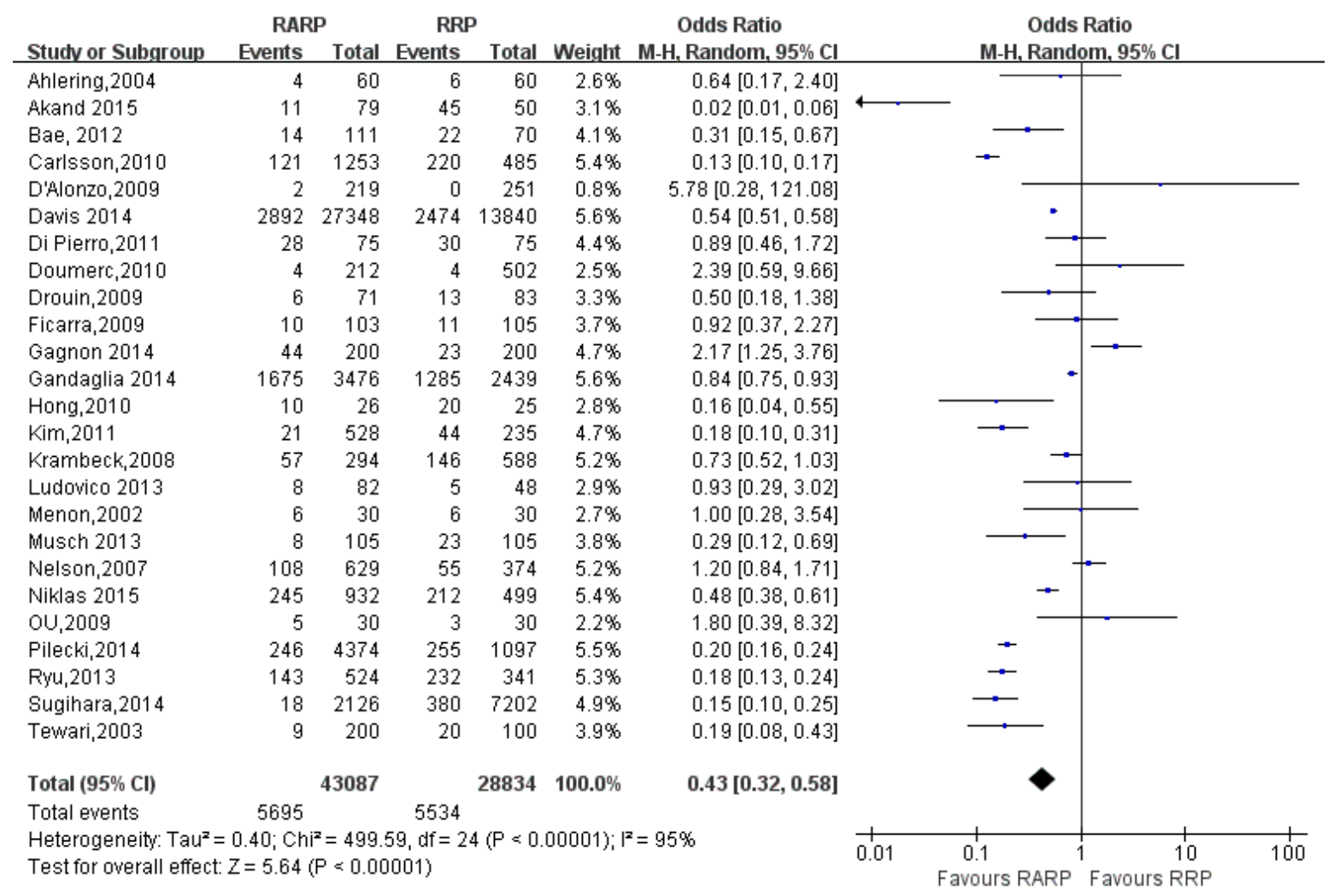

Figure 11: Forest plot and meta-analysis of overall complications between RARP and RRP. RARP = robot-assisted radical prostatectomy; RRP = retropubic radical prostatectomy. 
free survival than RRP(OR:1.33; 95\% CI: 1.01 to $1.76 ; P$ $=0.04$ ) (Figure 15). Pooling data from 7 studies[22, 35, $38,53,54,59,75]$ reported on readmission rate, the forest plot showed that RARP had a lower readmission rate than RRP(OR:0.83; 95\% CI: 0.74 to $0.94 ; P=0.002$ ) (Figure 16).

\section{Sensitivity analysis}

42 qualified studies with patients' baseline characteristic consistency(age, pre-PSA, BMI, prostate volume, $P>0.5)$ are analyzed by sensitivity analysis (Table 6). Compared with the original analysis, there was no change in the significance of any other outcomes except that readmission $\operatorname{rate}(P=0.002$ vs $P=0.13)$, and $\mathrm{BCR}$ for free $\operatorname{survival}(P=0.04$ vs. $P=0.55)$ were significantly different in sensitivity analysis. The method of sensitivity analysis can reduce the heterogeneity of studies to a certain extent.

\section{DISCUSSION}

The incidence of prostate cancer and its mortality is the first and the second common cancer in man, respectively[1]. Our results indicated that RARP seemed to have an younger age (WMD: $-1.00 ; P<0.001$ ), and to have the lower level of pre-PSA (WMD: $-0.93 ; P$ $<0.001)$ than RRP group, and that these differences are primarily due to surgeon's preference for surgical modality. Another reason is that the younger is more easier to choose new approach. However, there is no difference on BMI and prostate volume between the two groups. Sensitivity analysis showed that there was no change in the significance of any other outcomes except that readmission $\operatorname{rate}(P=0.002$ vs $P=0.13)$ and $\mathrm{BCR}$ for free survival $(P=0.04$ vs. $P=0.55)$.It demonstrated that selection bias of demographic and clinical data of patients is small between two groups.

Novara $G$ et al[6] evaluated oncologic outcomes of RARP and RRP, and the results indicated that RARP had less EBL and transfusion rate than RRP. Their results presented similar results and strengthened our results. The

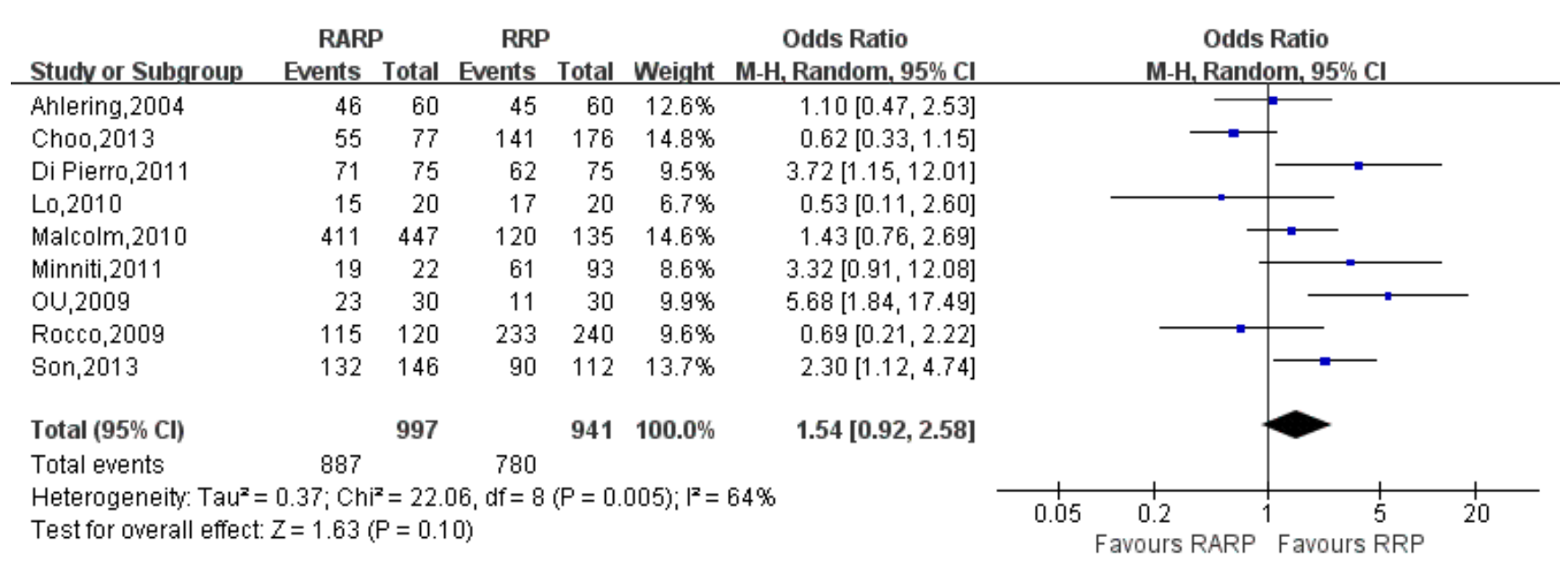

Figure 12: Forest plot and meta-analysis of 3-mo urinary continence rate between RARP and RRP. RARP $=$ robotassisted radical prostatectomy; $\mathrm{RRP}=$ retropubic radical prostatectomy.

\begin{tabular}{|c|c|c|c|c|c|c|c|c|c|c|}
\hline Study or Subgroup & $\begin{array}{l}\text { RAR } \\
\text { Events }\end{array}$ & Total & $\begin{array}{l}\text { RRP } \\
\text { Events }\end{array}$ & Total & Weight & $\begin{array}{c}\text { Odds Ratio } \\
\text { M-H, Random, } 95 \% \mathrm{Cl}\end{array}$ & & $\begin{array}{r}\text { Odds } \\
\text { M-H, Rando }\end{array}$ & $\begin{array}{l}\text { Ratio } \\
\text { lom, } 95 \% \mathrm{Cl}\end{array}$ & \\
\hline Choo,2013 & 7 & 41 & 3 & 55 & $15.6 \%$ & $3.57[0.86,14.76]$ & & & & \\
\hline Di Pierro,2011 & 25 & 37 & 12 & 49 & $18.9 \%$ & $6.42[2.49,16.57]$ & & & & \\
\hline Malcolm,2010 & 326 & 447 & 100 & 135 & $22.0 \%$ & $0.94[0.61,1.46]$ & & & . & \\
\hline Rocco,2009 & 37 & 120 & 43 & 240 & $21.6 \%$ & $2.04[1.23,3.40]$ & & & - & \\
\hline Ryu,2013 & 204 & 524 & 24 & 341 & $21.9 \%$ & $8.42[5.37,13.21]$ & & & $\rightarrow-$ & \\
\hline Total $(95 \% \mathrm{Cl})$ & & 1169 & & 820 & $100.0 \%$ & $3.19[1.19,8.56]$ & & & & \\
\hline Total events & 599 & & 182 & & & & & & & \\
\hline $\begin{array}{l}\text { Heterogeneity: } \text { Tau }^{2} \\
\text { Test for overall effec }\end{array}$ & $\begin{array}{l}1.11 ; \mathrm{Chi} \\
Z=2.30\end{array}$ & $\begin{array}{l}\mathrm{j}^{2}=51.9 \\
(P=0.0\end{array}$ & $\begin{array}{l}\text { 94, } d f=4 \\
\text { 2) }\end{array}$ & $(P<0$. & $00001) ; 1^{2}$ & $z=92 \%$ & 0.005 & $\begin{array}{c}0.1 \\
\text { Favours RARP }\end{array}$ & ${ }^{1} \frac{10}{\text { Favours RRP }}$ & $\frac{1}{200}$ \\
\hline
\end{tabular}

Figure 13: Forest plot and meta-analysis of 3-mo potent recovery rate between RARP and RRP. RARP = robot-assisted radical prostatectomy; RRP = retropubic radical prostatectomy. 
other analyzed parameters operative time and complication rate were similar. However, in our meta-analysis, RARP had longer operative time than RRP(WMD:39.85min, $P<0.001)$, which likely reflects the early learning curve with RARP. But the learning curve indicated that operative time was decreased with growing operative experience and it won't influenced operative outcomes[88].

With regard to the pathologic outcomes, patients underwent RARP had more pathological Gleason score $=$ 7 , less pathological Gleason score $\geq 8$, higher lymph node

\begin{tabular}{|c|c|c|c|c|c|c|c|c|c|c|}
\hline Studv or Subgroup & \multicolumn{2}{|c|}{ RARP } & \multicolumn{2}{|c|}{ RRP } & Weight & $\begin{array}{c}\text { Odds Ratio } \\
\text { M-H, Random, } 95 \% \mathrm{Cl}\end{array}$ & \multicolumn{4}{|c|}{$\begin{array}{c}\text { Odds Ratio } \\
\text { M-H, Random, } 95 \% \mathrm{Cl}\end{array}$} \\
\hline Di Pierro,2011 & 12 & 22 & 12 & 47 & $12.0 \%$ & $3.50[1.21,10.15]$ & & & & \\
\hline Ficarra,2009 & 52 & 64 & 20 & 41 & $13.7 \%$ & $4.55[1.89,10.94]$ & & & & \\
\hline Kim,2011 & 313 & 373 & 57 & 122 & $17.4 \%$ & $5.95[3.79,9.33]$ & & & $\rightarrow-$ & \\
\hline Krambeck,2008 & 142 & 203 & 262 & 417 & $18.0 \%$ & $1.38[0.96,1.97]$ & & & - & \\
\hline Ong 2015 & 321 & 639 & 351 & 731 & $18.8 \%$ & $1.09[0.88,1.35]$ & & & & \\
\hline OU,2009 & 6 & 16 & 1 & 2 & $3.4 \%$ & $0.60[0.03,11.47]$ & & & & \\
\hline Rocco,2009 & 48 & 78 & 88 & 214 & $16.7 \%$ & $2.29[1.35,3.90]$ & & & & \\
\hline Total $(95 \%$ Cl) & & 1395 & & 1574 & $100.0 \%$ & $2.37[1.30,4.33]$ & & & & \\
\hline Total events & 894 & & 791 & & & & & & & \\
\hline $\begin{array}{l}\text { Heterogeneity: Tau } \\
\text { Test for overall effect }\end{array}$ & $\begin{array}{l}0.49 ; \mathrm{Chi}^{-} \\
\mathrm{Z}=2.800\end{array}$ & $\begin{array}{l}P=55.4 \\
P=0.0\end{array}$ & $\begin{array}{l}43, d f=6 \\
05)\end{array}$ & $(P \leq 0$ & $00001) ; l^{2}$ & $=89 \%$ & 0.002 & $\begin{array}{ll}0.1 & 1 \\
\text { ours RARP }\end{array}$ & $\begin{array}{r}10 \\
\text { Favours R }\end{array}$ & $\overrightarrow{500}$ \\
\hline
\end{tabular}

Figure 14: Forest plot and meta-analysis of 12-mo potent recovery rate between $R A R P$ and $R R P$. RARP $=$ robot-assisted radical prostatectomy; $\mathrm{RRP}=$ retropubic radical prostatectomy.

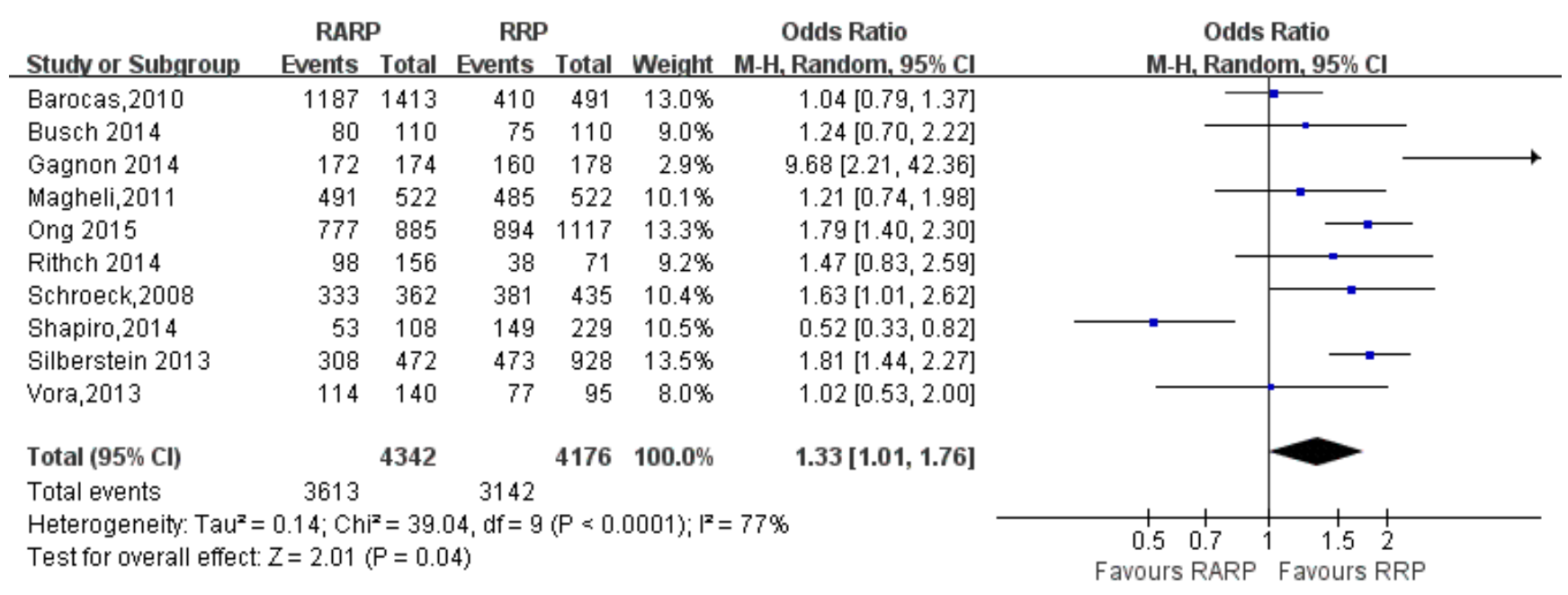

Figure 15: Forest plot and meta-analysis of BCR free survival rate between RARP and RRP. RARP $=$ robot-assisted radical prostatectomy; RRP = retropubic radical prostatectomy.

\begin{tabular}{|c|c|c|c|c|c|c|c|c|c|c|}
\hline Study or Subgroup & \multicolumn{2}{|c|}{ RARP } & \multicolumn{2}{|c|}{ RRP } & Weight & $\begin{array}{c}\text { Odds Ratio } \\
\text { M-H, Fixed, 95\% Cl }\end{array}$ & \multicolumn{4}{|c|}{$\begin{array}{c}\text { Odds Ratio } \\
\text { M-H, Fixed, 95\% Cl }\end{array}$} \\
\hline Chung,2012 & 10 & 274 & 190 & 1773 & $8.0 \%$ & $0.32[0.16,0.60]$ & & & & \\
\hline Gandaglia 2014 & 328 & 3476 & 236 & 2439 & $40.9 \%$ & $0.97[0.82,1.16]$ & & & & \\
\hline Hall 2014 & 2 & 100 & 19 & 100 & $3.0 \%$ & $0.09[0.02,0.38]$ & & & & \\
\hline Nelson, 2007 & 45 & 629 & 18 & 374 & $3.4 \%$ & $1.52[0.87,2.67]$ & & & & \\
\hline Niklas 2015 & 127 & 932 & 97 & 499 & $17.8 \%$ & $0.65[0.49,0.87]$ & & $\rightarrow$ & & \\
\hline Pilecki,2014 & 152 & 4374 & 60 & 1097 & $15.1 \%$ & $0.62[0.46,0.85]$ & & $\rightarrow$ & & \\
\hline Wallerstedt 2015 & 163 & 1847 & 57 & 778 & $11.9 \%$ & $1.22[0.89,1.68]$ & & & & \\
\hline Total $(95 \% \mathrm{Cl})$ & & 11632 & & 7060 & $100.0 \%$ & $0.83[0.74,0.94]$ & & $\boldsymbol{\Delta}$ & & \\
\hline Total events & 827 & & 677 & & & & & & & \\
\hline $\begin{array}{l}\text { Heterogeneity: } \mathrm{Chi}^{2}= \\
\text { Test for overall effec }\end{array}$ & $\begin{array}{l}36.82, \mathrm{df} \\
Z=3.09\end{array}$ & $\begin{array}{l}=6(\mathrm{P} s \\
P=0.00\end{array}$ & 0.00001 ) & $\left.\right|^{2}=$ & & & 0.05 & $\begin{array}{ll}0.2 & 1 \\
\text { vours RARP }\end{array}$ & 1 Favours $\mathrm{F}$ & $\begin{array}{l}5 \\
\mathrm{RRP}\end{array}$ \\
\hline
\end{tabular}

Figure 16: Forest plot and meta-analysis of readmission rate between RARP and RRP. RARP $=$ robot-assisted radical prostatectomy; RRP = retropubic radical prostatectomy. 
yield and fewer positive lymph node than RRP. However, the pathological $\mathrm{T}$ stage is no significant difference between the RARP and RRP group. LN yield was deemed an indicator of surgical quality by many surgeons[89]. RARP had a higher LN yield than RRP, the reason is that RARP has meticulous dissection with $3 \mathrm{D}$ vision and decrease the intraoperative blood loss which made the surgeon have more time and patience to acquiring higher LN yield. Therefore, the oncological outcomes in terms of PSM for T3 is higher in RARP than RRP. With the results that BCR free survival was higher in RARP than RRP. Some studies showed that the predictors of BCR were preoperative PSA. Gleason score, pathological stage, and PSM[49].

The experts suggested that patient outcomes and surgical approach were mainly required to improve for an accurate characterization of complications[90]. In our meta-analysis, Patients underwent RARP had fewer overall complications than RRP. The possible reason may be associated with lower EBL and less transfusion rate in RARP. Then a comprehensive classification of complications indicated that RRP had a higher incidence of rectal injury, pulmonary embolism, wound infections, bladder neck contracture, urinary retention, deep venous thrombosis, urinary leakage, lymphocele, and obturator nerve injury. There were no significant differences with regard to ileus and UTI between two groups.

Ficarra $\mathrm{V}$ et al[91] compared RARP with RRP with respect to 12-mo urinary continence. Their results indicated that RARP had a better 12-mo urinary continence recovery than $\mathrm{RRP}(\mathrm{OR}: 1.53 ; P=0.03)$. However, our results indicated that there were no statistical differences with regard to 3-mo and 12-mo urinary continence in two groups. The urinary continence receiving RP is influenced by preoperative patient characteristics, surgical techniques, and so on. Some studies found that patient age[92, 93], BMI[94], comorbidity index[95], and prostate volume $[96,97]$ were also the potential predictors of urinary incontinence. Increasing age, higher BMI, and large prostate volume are correlated with high risk of urinary incontinence who underwent RP. However, the 3- and 12-mo potent recovery rate of RARP was also better than RRP group, respectively. Analysis of predictors indicated that peroperative parameters might influence potency results. Relevant predictors included age at surgery, baseline erectile function, and comorbidities [98]. Other authors also confirmed that age and baseline erectile function of patients were affected the potent recovery in nerve-sparing RARP [93, 99].

On the other hand, we found better BCR free survival and lower readmission rate in RARP group in the original analysis. The reason is that meticulous dissection, lower blood loss and complications might provide patients better oncologic prognosis in RARP group. However, we observed no statistical differences between RARP and RRP in sensitivity analysis. Therefore, multicenter, large sample, long follow-up RCTs are required to prove our findings.

Nevertheless, there were several limitations when analyzing and interpreting results in our meta-analysis. The major limitation is lack of well designed prospective, randomized control studies in our meta-analysis. Secondly, there existed heterogeneities of studies, especially in the comparing of the continuous data such as the length of hospital stay, operative time. whereas these parameters were influenced by the heterogeneities of patients' conditions, surgeon's surgical skills and the sample size of studies. In addition, short follow-up duration may have an influence on the confidence of outcomes. In the future, well-designed, prospective, multicenter randomized control studies are required to help us better demonstrate the advantages as well as drawbacks of this novel approach.

\section{MATERIALS AND METHODS}

\section{Literature search strategy}

To update previous systematic review [5-7, 91, 98, $100,101]$, a systematic review of published literature was performed according to the Cochrane Handbook recommendations[102]. No ethic issues get involved in this article. A systematic dissertion was conducted using Medline, Embase, Pubmed, CNKI, and all relevant studies had been identified by the Cochrane Library. The following key words were used: "comparative studies", "retropubic", "open", "radical prostatectomy ", "Da Vinci", "robot-assisted", and "prostate cancer".

\section{Data extraction and outcomes of interest}

Two of the authors(JKH and TK) extracted data from the selected studies including: author identification, country, publication year, study design, age, No. of patients, operative approaches were mentioned previously, and results of intervention. All disagreements about eligibility were reached a consensus through authors discussion. Perioperative outcomes including operative time, EBL, LOS, overall complications, and oncological outcomes were compared between the two methods from all the studies that were finally selected. Overall complications were graded on the basis of the ClavienDindo system[103].

\section{Inclusion criteria and exclusion criteria}

Studies should satisfy the following requirements: (1) to compare RARP with RRP, (2) to display on outcome of two approaches, (3) to document the surgery 
as RARP or RRP, (4) to clearly document indications for prostatectomy with prostate cancer. Studies will be excluded if (1) the study was not satisfied inclusion criteria or (2) the outcomes of literature were not mentioned or the parameters were impossible to analysis for either RARP or RRP from the published findings and (3) studies focusing on pure robot surgery system and/or on singlesite techniques.

\section{Study quality assessment and level of evidence}

In accordance with the criteria of Centre for Evidence-Based Medicine in Oxford, we evaluated the level of evidence(LOE) of included sixteen studies. The Jaded Score was applied to evaluated the methodological quality of RCTs[104]. The Newcastle-Ottawa Scale(NOS) was applied to assessed the methodological quality of nonRCTs observational studies $[86,105]$. Two authors(JKH and TK) evaluated the quality of the studies and discrepancies were rechecked by the third reviewer(CZQ) and consensus was achieved by discussion.

\section{Statistical analysis}

All meta-analysis were conducted by Review Manger 5.3(Cochrane Collaboration, Oxford, UK). Continuous and dichotomous variables were calculated by weighted mean differences (WMDs) and odds ratios(ORs). All analysis results were reported with $95 \%$ confidence intervals(CIs). $\mathrm{I}^{2}$ test and chi-square-based Q test were applied to evaluated the quantity of heterogeneity, and when $\mathrm{I}^{2}>50 \%$, the evidence was considered to have substantial heterogeneity, the random- effects(RE) model would be applied, otherwise, the fixed effects(FE) model was applied. The presence of publication bias was evaluated by Egger's test and funnel plot. Sensitivity analysis was used to estimate the influence of studies with a high risk of bias on the overall effect.

\section{CONFLICTS OF INTEREST}

The authors have no conflict of interest to disclose.

\section{REFERENCES}

1. Siegel RL, Miller KD, Jemal A. Cancer statistics, 2015. CA Cancer J Clin. 2015; 65: 5-29. doi: 10.3322/caac.21254.

2. Heidenreich A, Bellmunt J, Bolla M, Joniau S, Mason M, Matveev V, Mottet N, Schmid HP, van der Kwast T, Wiegel T, Zattoni F. EAU guidelines on prostate cancer. Part 1: screening, diagnosis, and treatment of clinically localised disease. Eur Urol. 2011; 59: 61-71. doi: 10.1016/j. eururo.2010.10.039.

3. Gratzke C, Dovey Z, Novara G, Geurts N, De Groote
R, Schatteman P, de Naeyer G, Gandaglia G, Mottrie A. Early Catheter Removal after Robot-assisted Radical Prostatectomy: Surgical Technique and Outcomes for the Aalst Technique (ECaRemA Study). Eur Urol. 2015. doi: 10.1016/j.eururo.2015.09.052.

4. Allan C, Ilic D. Laparoscopic versus Robotic-Assisted Radical Prostatectomy for the Treatment of Localised Prostate Cancer: A Systematic Review. Urol Int. 2015. doi: $10.1159 / 000435861$.

5. Tewari A, Sooriakumaran P, Bloch DA, Seshadri-Kreaden U, Hebert AE, Wiklund P. Positive surgical margin and perioperative complication rates of primary surgical treatments for prostate cancer: a systematic review and meta-analysis comparing retropubic, laparoscopic, and robotic prostatectomy. Eur Urol. 2012; 62: 1-15. doi: 10.1016/j.eururo.2012.02.029.

6. Novara G, Ficarra V, Mocellin S, Ahlering TE, Carroll PR, Graefen M, Guazzoni G, Menon M, Patel VR, Shariat SF, Tewari AK, Van Poppel H, Zattoni F, et al. Systematic review and meta-analysis of studies reporting oncologic outcome after robot-assisted radical prostatectomy. Eur Urol. 2012; 62: 382-404. doi: 10.1016/j.eururo.2012.05.047.

7. Novara G, Ficarra V, Rosen RC, Artibani W, Costello A, Eastham JA, Graefen M, Guazzoni G, Shariat SF, Stolzenburg JU, Van Poppel H, Zattoni F, Montorsi F, et al. Systematic review and meta-analysis of perioperative outcomes and complications after robot-assisted radical prostatectomy. Eur Urol. 2012; 62: 431-52. doi: 10.1016/j. eururo.2012.05.044.

8. Ball AJ GB, Fabrizio MD, Davis JW, Given RW, Lynch DF, Shaves M, Schellhammer PF. Prospective longitudinal comparative study of early health-related quality-of-life outcomes in patients undergoing surgical treatment for localized prostate cancer: a short-term evaluation of five approaches from a single institution. J Endourol. 2006; 20: 723-31. doi: 10.1089/end.2006.20.723.

9. Ahlering TE, Woo D, Eichel L, Lee DI, Edwards R, Skarecky DW. Robot-assisted versus open radical prostatectomy: a comparison of one surgeon's outcomes. Urology. 2004; 63: 819-22. doi: 10.1016/j. urology.2004.01.038.

10. Alemozaffar M, Sanda M, Yecies D, Mucci LA, Stampfer MJ, Kenfield SA. Benchmarks for operative outcomes of robotic and open radical prostatectomy: results from the Health Professionals Follow-up Study. Eur Urol. 2015; 67: 432-8. doi: 10.1016/j.eururo.2014.01.039.

11. Bae JJ, Choi SH, Kwon TG, Kim TH. Advantages of robot-assisted laparoscopic radical prostatectomy in obese patients: comparison with the open procedure. Korean $\mathrm{J}$ Urol. 2012; 53: 536-40. doi: 10.4111/kju.2012.53.8.536.

12. Barocas DA, Salem S, Kordan Y, Herrell SD, Chang SS, Clark PE, Davis R, Baumgartner R, Phillips S, Cookson MS, Smith JA, Jr. Robotic assisted laparoscopic prostatectomy versus radical retropubic prostatectomy for clinically localized prostate cancer: comparison of short- 
term biochemical recurrence-free survival. J Urol. 2010; 183: 990-6. doi: 10.1016/j.juro.2009.11.017.

13. Bolenz C, Gupta A, Hotze T, Ho R, Cadeddu JA, Roehrborn $\mathrm{CG}$, Lotan Y. Cost comparison of robotic, laparoscopic, and open radical prostatectomy for prostate cancer. Eur Urol. 2010; 57: 453-8. doi: 10.1016/j.eururo.2009.11.008.

14. Breyer BN, Davis CB, Cowan JE, Kane CJ, Carroll PR. Incidence of bladder neck contracture after robot-assisted laparoscopic and open radical prostatectomy. BJU Int. 2010; 106: 1734-8. doi: 10.1111/j.1464-410X.2010.09333.x.

15. Busch J, Gonzalgo ML, Leva N, Ferrari M, Cash H, Kempkensteffen C, Hinz S, Miller K, Magheli A. Matched comparison of robot-assisted, laparoscopic and open radical prostatectomy regarding pathologic and oncologic outcomes in obese patients. World J Urol. 2015; 33: 397 402. doi: 10.1007/s00345-014-1326-1.

16. Busch J, Magheli A, Leva N, Hinz S, Ferrari M, Friedersdorff F, Fuller TF, Miller K, Gonzalgo ML. Matched comparison of outcomes following open and minimally invasive radical prostatectomy for high-risk patients. World J Urol. 2014; 32: 1411-6. doi: 10.1007/ s00345-014-1270-0.

17. Carlsson S, Nilsson AE, Schumacher MC, Jonsson MN, Volz DS, Steineck G, Wiklund PN. Surgery-related complications in 1253 robot-assisted and 485 open retropubic radical prostatectomies at the Karolinska University Hospital, Sweden. Urology. 2010; 75: 1092-7. doi: 10.1016/j.urology.2009.09.075.

18. Chan RC, Barocas DA, Chang SS, Herrell SD, Clark PE, Baumgartner R, Smith JA, Cookson MS. Effect of a large prostate gland on open and robotically assisted laparoscopic radical prostatectomy. BJU Int. 2008; 101: 1140-4. doi: 10.1111/j.1464-410X.2007.07428.x.

19. Chino J, Schroeck FR, Sun L, Lee WR, Albala DM, Moul JW, Koontz BF. Robot-assisted laparoscopic prostatectomy is not associated with early postoperative radiation therapy. BJU Int. 2009; 104: 1496-500. doi: 10.1111/j.1464410X.2009.08588.x.

20. Choi D, Kim D, Kyung YS, Lim JH, Song SH, You D, Jeong IG, Kim CS. Clinical experience with limited lymph node dissection for prostate cancer in Korea: single center comparison of 247 open and 354 robot-assisted laparoscopic radical prostatectomy series. Korean J Urol. 2012; 53: 75560. doi: 10.4111/kju.2012.53.11.755.

21. Choo MS, Choi WS, Cho SY, Ku JH, Kim HH, Kwak C. Impact of prostate volume on oncological and functional outcomes after radical prostatectomy: robot-assisted laparoscopic versus open retropubic. Korean J Urol. 2013; 54: 15-21. doi: 10.4111/kju.2013.54.1.15.

22. Chung SD, Kelle JJ, Huang CY, Chen YH, Lin HC. Comparison of 90-day re-admission rates between open retropubic radical prostatectomy (RRP), laparoscopic RP (LRP) and robot-assisted laparoscopic prostatectomy (RALP). BJU Int. 2012; 110: E966-71. doi: 10.1111/j.1464410X.2012.11183.x.
23. D'Alonzo RC, Gan TJ, Moul JW, Albala DM, Polascik TJ, Robertson CN, Sun L, Dahm P, Habib AS. A retrospective comparison of anesthetic management of robot-assisted laparoscopic radical prostatectomy versus radical retropubic prostatectomy. J Clin Anesth. 2009; 21: 322-8. doi: 10.1016/j.jclinane.2008.09.005.

24. Davis JW, Kreaden US, Gabbert J, Thomas R. Learning curve assessment of robot-assisted radical prostatectomy compared with open-surgery controls from the premier perspective database. J Endourol. 2014; 28: 560-6. doi: 10.1089/end.2013.0534.

25. Davison BJ, Matthew A, Gardner AM. Prospective comparison of the impact of robotic-assisted laparoscopic radical prostatectomy versus open radical prostatectomy on health-related quality of life and decision regret. Can Urol Assoc J. 2014; 8: E68-72. doi: 10.5489/cuaj.480.

26. Di Pierro GB, Baumeister P, Stucki P, Beatrice J, Danuser $\mathrm{H}$, Mattei A. A prospective trial comparing consecutive series of open retropubic and robot-assisted laparoscopic radical prostatectomy in a centre with a limited caseload. Eur Urol. 2011; 59: 1-6. doi: 10.1016/j.eururo.2010.10.026.

27. Doumerc N, Yuen C, Savdie R, Rahman MB, Rasiah KK, Pe Benito R, Delprado W, Matthews J, Haynes AM, Stricker PD. Should experienced open prostatic surgeons convert to robotic surgery? The real learning curve for one surgeon over 3 years. BJU Int. 2010; 106: 378-84. doi: 10.1111/j.1464-410X.2009.09158.x.

28. Drouin SJ, Comperat E, Varinot J, Vaessen C, Bitker MO, Chartier-Kastler E, Mozer P, Shariat SF, Cussenot O, Roupret M. The surgical approach can be determined from the pathological specimen obtained after open or robotassisted laparoscopic radical prostatectomy. World J Urol. 2014; 32: 489-93. doi: 10.1007/s00345-013-1107-2.

29. Drouin SJ, Vaessen C, Hupertan V, Comperat E, Misrai V, Haertig A, Bitker MO, Chartier-Kastler E, Richard F, Roupret M. Comparison of mid-term carcinologic control obtained after open, laparoscopic, and robot-assisted radical prostatectomy for localized prostate cancer. World J Urol. 2009; 27: 599-605. doi: 10.1007/s00345-009-0379-z.

30. Farnham SB, Webster TM, Herrell SD, Smith JA, Jr. Intraoperative blood loss and transfusion requirements for robotic-assisted radical prostatectomy versus radical retropubic prostatectomy. Urology. 2006; 67: 360-3. doi: 10.1016/j.urology.2005.08.029.

31. Ficarra V, Novara G, Artibani W, Cestari A, Galfano A, Graefen M, Guazzoni G, Guillonneau B, Menon M, Montorsi F, Patel V, Rassweiler J, Van Poppel H. Retropubic, laparoscopic, and robot-assisted radical prostatectomy: a systematic review and cumulative analysis of comparative studies. Eur Urol. 2009; 55: 1037-63. doi: 10.1016/j.eururo.2009.01.036.

32. Fracalanza S, Ficarra V, Cavalleri S, Galfano A, Novara G, Mangano A, Plebani M, Artibani W. Is robotically assisted laparoscopic radical prostatectomy less invasive than retropubic radical prostatectomy? 
Results from a prospective, unrandomized, comparative study. BJU Int. 2008; 101: 1145-9. doi: 10.1111/j.1464410X.2008.07513.x.

33. Froehner M, Koch R, Leike S, Novotny V, Twelker L, Wirth MP. Urinary tract-related quality of life after radical prostatectomy: open retropubic versus robot-assisted laparoscopic approach. Urol Int. 2013; 90: 36-40. doi: $10.1159 / 000345320$.

34. Gagnon LO, Goldenberg SL, Lynch K, Hurtado A, Gleave ME. Comparison of open and robotic-assisted prostatectomy: The University of British Columbia experience. Can Urol Assoc J. 2014; 8: 92-7. doi: 10.5489/ cuaj.1707.

35. Gandaglia G, Sammon JD, Chang SL, Choueiri TK, Hu JC, Karakiewicz PI, Kibel AS, Kim SP, Konijeti R, Montorsi F, Nguyen PL, Sukumar S, Menon M, et al. Comparative effectiveness of robot-assisted and open radical prostatectomy in the postdissemination era. J Clin Oncol. 2014; 32: 1419-26. doi: 10.1200/JCO.2013.53.5096.

36. Geraerts I, Van Poppel H, Devoogdt N, Van Cleynenbreugel B, Joniau S, Van Kampen M. Prospective evaluation of urinary incontinence, voiding symptoms and quality of life after open and robot-assisted radical prostatectomy. BJU Int. 2013; 112: 936-43. doi: 10.1111/bju.12258.

37. Haglind E, Carlsson S, Stranne J, Wallerstedt A, Wilderang U, Thorsteinsdottir T, Lagerkvist M, Damber JE, Bjartell A, Hugosson J, Wiklund P, Steineck G, committee Ls. Urinary Incontinence and Erectile Dysfunction After Robotic Versus Open Radical Prostatectomy: A Prospective, Controlled, Nonrandomised Trial. Eur Urol. 2015; 68: 216-25. doi: 10.1016/j.eururo.2015.02.029.

38. Hall RM, Linklater N, Coughlin G. Robotic and open radical prostatectomy in the public health sector: cost comparison. ANZ J Surg. 2014; 84: 477-80. doi: 10.1111/ ans.12097.

39. Harty NJ, Kozinn SI, Canes D, Sorcini A, Moinzadeh A. Comparison of positive surgical margin rates in high risk prostate cancer: open versus minimally invasive radical prostatectomy. Int Braz J Urol. 2013; 39: 639-46; discussion 47-8. doi: 10.1590/S1677-5538.IBJU.2013.05.05.

40. Hong JY, Kim JY, Choi YD, Rha KH, Yoon SJ, Kil HK. Incidence of venous gas embolism during robotic-assisted laparoscopic radical prostatectomy is lower than that during radical retropubic prostatectomy. Br J Anaesth. 2010; 105: 777-81. doi: 10.1093/bja/aeq247.

41. Hu JC, Gandaglia G, Karakiewicz PI, Nguyen PL, Trinh QD, Shih YC, Abdollah F, Chamie K, Wright JL, Ganz PA, Sun M. Comparative effectiveness of robot-assisted versus open radical prostatectomy cancer control. Eur Urol. 2014; 66: 666-72. doi: 10.1016/j.eururo.2014.02.015.

42. Kim SC, Song C, Kim W, Kang T, Park J, Jeong IG, Lee S, Cho YM, Ahn H. Factors determining functional outcomes after radical prostatectomy: robot-assisted versus retropubic. Eur Urol. 2011; 60: 413-9. doi: 10.1016/j. eururo.2011.05.011
43. Koo KC, Tuliao P, Yoon YE, Chung BH, Hong SJ, Yang $\mathrm{SC}$, Rha KH. Robot-assisted radical prostatectomy in the Korean population: a 5-year propensity-score matched comparative analysis versus open radical prostatectomy. Int J Urol. 2014; 21: 781-5. doi: 10.1111/iju.12447.

44. Kordan Y, Barocas DA, Altamar HO, Clark PE, Chang SS, Davis R, Herrell SD, Baumgartner R, Mishra V, Chan RC, Smith JA, Jr., Cookson MS. Comparison of transfusion requirements between open and robotic-assisted laparoscopic radical prostatectomy. BJU Int. 2010; 106: 1036-40. doi: 10.1111/j.1464-410X.2010.09233.x.

45. Korets R, Weinberg AC, Alberts BD, Woldu SL, Mann MJ, Badani KK. Utilization and timing of blood transfusions following open and robot-assisted radical prostatectomy. J Endourol. 2014; 28: 1418-23. doi: 10.1089/end.2014.0225.

46. Krambeck AE, DiMarco DS, Rangel LJ, Bergstralh EJ, Myers RP, Blute ML, Gettman MT. Radical prostatectomy for prostatic adenocarcinoma: a matched comparison of open retropubic and robot-assisted techniques. BJU Int. 2009; 103: 448-53. doi: 10.1111/j.1464410X.2008.08012.x.

47. Laurila TA, Huang W, Jarrard DF. Robotic-assisted laparoscopic and radical retropubic prostatectomy generate similar positive margin rates in low and intermediate risk patients. Urol Oncol. 2009; 27: 529-33. doi: 10.1016/j. urolonc.2008.05.001.

48. Ludovico GM, Dachille G, Pagliarulo G, D'Elia C, Mondaini N, Gacci M, Detti B, Malossini G, Bartoletti $\mathrm{R}$, Cai T. Bilateral nerve sparing robotic-assisted radical prostatectomy is associated with faster continence recovery but not with erectile function recovery compared with retropubic open prostatectomy: the need for accurate selection of patients. Oncol Rep. 2013; 29: 2445-50. doi: 10.3892/or.2013.2365.

49. Magheli A, Gonzalgo ML, Su LM, Guzzo TJ, Netto G, Humphreys EB, Han M, Partin AW, Pavlovich CP. Impact of surgical technique (open vs laparoscopic vs roboticassisted) on pathological and biochemical outcomes following radical prostatectomy: an analysis using propensity score matching. BJU Int. 2011; 107: 1956-62. doi: 10.1111/j.1464-410X.2010.09795.x.

50. Malcolm JB, Fabrizio MD, Barone BB, Given RW, Lance RS, Lynch DF, Davis JW, Shaves ME, Schellhammer PF. Quality of life after open or robotic prostatectomy, cryoablation or brachytherapy for localized prostate cancer. J Urol. 2010; 183: 1822-8. doi: 10.1016/j.juro.2009.12.102.

51. Miller J, Smith A, Kouba E, Wallen E, Pruthi RS. Prospective evaluation of short-term impact and recovery of health related quality of life in men undergoing robotic assisted laparoscopic radical prostatectomy versus open radical prostatectomy. J Urol. 2007; 178: 854-8; discussion 9. doi: 10.1016/j.juro.2007.05.051.

52. Musch M, Roggenbuck U, Klevecka V, Loewen H, Janowski M, Davoudi Y, Kroepfl D. Does changeover by an experienced open prostatic surgeon from open retropubic 
to robot-assisted laparoscopic prostatectomy mean a step forward or backward? ISRN Oncol. 2013; 2013: 768647. doi: 10.1155/2013/768647.

53. Nelson B, Kaufman M, Broughton G, Cookson MS, Chang SS, Herrell SD, Baumgartner RG, Smith JA, Jr. Comparison of length of hospital stay between radical retropubic prostatectomy and robotic assisted laparoscopic prostatectomy. J Urol. 2007; 177: 929-31. doi: 10.1016/j. juro.2006.10.070.

54. Niklas C, Saar M, Berg B, Steiner K, Janssen M, Siemer S, Stockle M, Ohlmann CH. da Vinci and Open Radical Prostatectomy: Comparison of Clinical Outcomes and Analysis of Insurance Costs. Urol Int. 2015. doi: $10.1159 / 000431104$.

55. O’Neil B, Koyama T, Alvarez J, Conwill RM, Albertsen PC, Cooperberg MR, Goodman M, Greenfield S, Hamilton AS, Hoffman KE, Hoffman RM, Kaplan SH, Stanford JL, et al. The Comparative Harms of Open and Robotic Prostatectomy in Population Based Samples. J Urol. 2015. doi: 10.1016/j.juro.2015.08.092.

56. Ong WL, Evans SM, Spelman T, Kearns PA, Murphy DG, Millar JL. Comparison of oncological and health related quality of life (HRQOL) outcomes between open (ORP) and robotic-assisted radical prostatectomy (RARP) for localized prostate cancer - findings from the populationbased Victorian Prostate Cancer Registry (PCR). BJU Int. 2015. doi: 10.1111/bju.13380.

57. Park J, Yoo DS, Song C, Park S, Park S, Kim SC, Cho Y, Ahn H. Comparison of oncological outcomes between retropubic radical prostatectomy and robot-assisted radical prostatectomy: an analysis stratified by surgical experience. World J Urol. 2014; 32: 193-9. doi: 10.1007/s00345-0131168-2.

58. Pierorazio PM, Mullins JK, Eifler JB, Voth K, Hyams ES, Han M, Pavlovich CP, Bivalacqua TJ, Partin AW, Allaf ME, Schaeffer EM. Contemporaneous comparison of open $v s$ minimally-invasive radical prostatectomy for high-risk prostate cancer. BJU Int. 2013; 112: 751-7. doi: 10.1111/j.1464-410X.2012.11757.x.

59. Pilecki MA, McGuire BB, Jain U, Kim JY, Nadler RB. National multi-institutional comparison of 30-day postoperative complication and readmission rates between open retropubic radical prostatectomy and robot-assisted laparoscopic prostatectomy using NSQIP. J Endourol. 2014; 28: 430-6. doi: 10.1089/end.2013.0656.

60. Porcaro AB, Molinari A, Terrin A, De Luyk N, Baldassarre R, Brunelli M, Cavalleri S, Cerruto MA, Gelati M, Salvagno GL, Guidi GC, Artibani W. Robotic-assisted radical prostatectomy is less stressful than the open approach: results of a contemporary prospective study evaluating pathophysiology of cortisol stress-related kinetics in prostate cancer surgery. J Robot Surg. 2015; 9: 249-55. doi: 10.1007/s11701-015-0522-3.

61. Ritch CR, You C, May AT, Herrell SD, Clark PE, Penson DF, Chang SS, Cookson MS, Smith JA, Jr., Barocas DA.
Biochemical recurrence-free survival after robotic-assisted laparoscopic $v s$ open radical prostatectomy for intermediateand high-risk prostate cancer. Urology. 2014; 83: 1309-15. doi: 10.1016/j.urology.2014.02.023.

62. Rocco B, Matei DV, Melegari S, Ospina JC, Mazzoleni F, Errico G, Mastropasqua M, Santoro L, Detti S, de Cobelli O. Robotic $v s$ open prostatectomy in a laparoscopically naive centre: a matched-pair analysis. BJU Int. 2009; 104: 991-5. doi: 10.1111/j.1464-410X.2009.08532.x.

63. Rush S, Alibhai SM, Xu L, Xu W, Louis AS, Matthew AG, Nesbitt M, Finelli A, Fleshner NE, Hamilton RJ, Kulkarni G, Zlotta A, Jewett MA, et al. Health-related quality of life in robotic versus open radical prostatectomy. Can Urol Assoc J. 2015; 9: 179-87. doi: 10.5489/cuaj.2618.

64. Ryu J, Kwon T, Kyung YS, Hong S, You D, Jeong IG, Kim CS. Retropubic versus robot-assisted laparoscopic prostatectomy for prostate cancer: a comparative study of postoperative complications. Korean J Urol. 2013; 54: 75661. doi: 10.4111/kju.2013.54.11.756.

65. Schroeck FR, Sun L, Freedland SJ, Albala DM, Mouraviev V, Polascik TJ, Moul JW. Comparison of prostate-specific antigen recurrence-free survival in a contemporary cohort of patients undergoing either radical retropubic or robotassisted laparoscopic radical prostatectomy. BJU Int. 2008; 102: 28-32. doi: 10.1111/j.1464-410X.2008.07607.x.

66. Shapiro EY, Scarberry K, Patel T, Bergman A, Ahn JJ, Sahi N, RoyChoudhury A, Deutch I, McKiernan JM, Benson MC, Badani KK. Comparison of robot-assisted and open retropubic radical prostatectomy for risk of biochemical progression in men with positive surgical margins. J Endourol. 2014; 28: 208-13. doi: 10.1089/end.2013.0393.

67. Silberstein JL, Su D, Glickman L, Kent M, Keren-Paz G, Vickers AJ, Coleman JA, Eastham JA, Scardino PT, Laudone VP. A case-mix-adjusted comparison of early oncological outcomes of open and robotic prostatectomy performed by experienced high volume surgeons. BJU Int. 2013; 111: 206-12. doi: 10.1111/j.1464410X.2012.11638.x.

68. Silberstein JL, Vickers AJ, Power NE, Parra RO, Coleman JA, Pinochet R, Touijer KA, Scardino PT, Eastham JA, Laudone VP. Pelvic lymph node dissection for patients with elevated risk of lymph node invasion during radical prostatectomy: comparison of open, laparoscopic and robotassisted procedures. J Endourol. 2012; 26: 748-53. doi: 10.1089/end.2011.0266.

69. Smith JA, Jr., Chan RC, Chang SS, Herrell SD, Clark PE, Baumgartner R, Cookson MS. A comparison of the incidence and location of positive surgical margins in robotic assisted laparoscopic radical prostatectomy and open retropubic radical prostatectomy. J Urol. 2007; 178: 2385-9; discussion 9-90. doi: 10.1016/j.juro.2007.08.008.

70. Son SJ, Lee SC, Jeong CW, Jeong SJ, Byun SS, Lee SE. Comparison of continence recovery between robot-assisted laparoscopic prostatectomy and open radical retropubic prostatectomy: a single surgeon experience. Korean J Urol. 
2013; 54: 598-602. doi: 10.4111/kju.2013.54.9.598.

71. Stranne J, Johansson E, Nilsson A, Bill-Axelson A, Carlsson S, Holmberg L, Johansson JE, Nyberg T, Ruutu M, Wiklund NP, Steineck G. Inguinal hernia after radical prostatectomy for prostate cancer: results from a randomized setting and a nonrandomized setting. Eur Urol. 2010; 58: 719-26. doi: 10.1016/j.eururo.2010.08.006.

72. Sugihara T, Yasunaga H, Horiguchi H, Matsui H, Fujimura T, Nishimatsu H, Fukuhara H, Kume H, Changhong Y, Kattan MW, Fushimi K, Homma Y. Robot-assisted versus other types of radical prostatectomy: population-based safety and cost comparison in Japan, 2012-2013. Cancer Sci. 2014; 105: 1421-6. doi: 10.1111/cas.12523.

73. Tewari A, Srivasatava A, Menon M. A prospective comparison of radical retropubic and robot-assisted prostatectomy: experience in one institution. BJU International. 2003; 92: 205-10. doi: 10.1046/j.1464410X.2003.04311.X.

74. Vora AA, Marchalik D, Kowalczyk KJ, Nissim H, Bandi G, McGeagh KG, Lynch JH, Ghasemian SR, Verghese M, Venkatesan K, Borges P, Uchio EM, Hwang JJ. Robotic-assisted prostatectomy and open radical retropubic prostatectomy for locally-advanced prostate cancer: multiinstitution comparison of oncologic outcomes. Prostate Int. 2013; 1: 31-6. doi: 10.12954/PI.12001.

75. Wallerstedt A, Tyritzis SI, Thorsteinsdottir T, Carlsson S, Stranne J, Gustafsson O, Hugosson J, Bjartell A, Wilderang U, Wiklund NP, Steineck G, Haglind E, committee Ls. Short-term results after robot-assisted laparoscopic radical prostatectomy compared to open radical prostatectomy. Eur Urol. 2015; 67: 660-70. doi: 10.1016/j.eururo.2014.09.036.

76. White MA, De Haan AP, Stephens DD, Maatman TK, Maatman TJ. Comparative analysis of surgical margins between radical retropubic prostatectomy and RALP: are patients sacrificed during initiation of robotics program? Urology. 2009; 73: 567-71. doi: 10.1016/j. urology.2008.11.011.

77. Williams SB, Chen MH, D'Amico AV, Weinberg AC, Kacker R, Hirsch MS, Richie JP, Hu JC. Radical retropubic prostatectomy and robotic-assisted laparoscopic prostatectomy: likelihood of positive surgical margin(s). Urology. 2010; 76: 1097-101. doi: 10.1016/j. urology.2009.11.079.

78. Wood DP, Schulte R, Dunn RL, Hollenbeck BK, Saur R, Wolf JS, Jr., Montie JE. Short-term health outcome differences between robotic and conventional radical prostatectomy. Urology. 2007; 70: 945-9. doi: 10.1016/j. urology.2007.06.1120.

79. Yi JS, Kwak C, Kim HH, Ku JH. Surgical clip-related complications after radical prostatectomy. Korean J Urol. 2010; 51: 683-7. doi: 10.4111/kju.2010.51.10.683.

80. Akand M CO, Avci E, Duman I, Erdogru T. Open, laparoscopic and robot-assisted laparoscopic radical prostatectomy: comparative analysis of operative and pathologic outcomes for three techniques with a single surgeon's experience. Eur Rev Med Pharmacol Sci. 2015; 19: 525-31. doi:

81. Lo KL NC, Lam CN, Hou SS, To KF, Yip SK. Short-term outcome of patients with robot-assisted versus open radical prostatectomy: for localised carcinoma of prostate. Hong Kong Med J. doi:

82. Menon M TA, Baize B, Guillonneau B, Vallancien G. Prospective comparison of radical retropubic prostatectomy and robot-assisted anatomic prostatectomy: the Vattikuti Urology Institute experience. Urology. 2002; 60: 864-8.

83. Minniti D, Chiado Piat S, Di Novi C. Robot-assisted versus open radical prostatectomy: an evidence-based comparison. Technol Health Care. 2011; 19:331-339. doi: 10.3233/THC2011-0635.

84. Ou YC, Yang CR, Wang J, Cheng CL, Patel VR. Comparison of robotic-assisted versus retropubic radical prostatectomy performed by a single surgeon. Anticancer Res. 2009; 29. doi:

85. Truesdale MD, Lee DJ, Cheetham PJ, Hruby GW, Turk AT, Badani KK. Assessment of lymph node yield after pelvic lymph node dissection in men with prostate cancer: a comparison between robot-assisted radical prostatectomy and open radical prostatectomy in the modern era. J Endourol. 2010; 24: 1055-60. doi: 10.1089/end.2010.0128.

86. Feng MX, Hong JX, Wang Q, Fan YY, Yuan CT, Lei XH, Zhu M, Qin A, Chen HX, Hong D. Dihydroartemisinin prevents breast cancer-induced osteolysis via inhibiting both breast caner cells and osteoclasts. Sci Rep. 2016; 6: 19074. doi: 10.1038/srep19074.

87. Clark HD, Wells GA, Huët C, McAlister FA, Salmi LR, Fergusson D, Laupacis A. Assessing the Quality of Randomized Trials:Reliability of the Jadad Scale. 1999. doi: 10.1590/s1677-55382011000200002.

88. Vasdev N, Bishop C, Kass-Iliyya A, Hamid S, McNicholas TA, Prasad V, Mohan SG, Lane T, Boustead G, Adshead JM. Developing a robotic prostatectomy service and a robotic fellowship programme - defining the learning curve. Curr Urol. 2013; 7: 136-44. doi: 10.1159/000356266.

89. Heidenreich A PD. Extended pelvic lymphadenectomy in prostate cancer: Practice makes perfect. Can Urol Assoc J. 2015; 9: E232-3. doi: 10.5489/cuaj.2877.

90. Donat SM. Standards for surgical complication reporting in urologic oncology: time for a change. Urology. 2007; 69: 221-5. doi: 10.1016/j.urology.2006.09.056.

91. Ficarra V, Novara G, Rosen RC, Artibani W, Carroll PR, Costello A, Menon M, Montorsi F, Patel VR, Stolzenburg JU, Van der Poel H, Wilson TG, Zattoni F, et al. Systematic review and meta-analysis of studies reporting urinary continence recovery after robot-assisted radical prostatectomy. Eur Urol. 2012; 62: 405-17. doi: 10.1016/j. eururo.2012.05.045.

92. Patel VR, Samavedi S, Bates AS, Kumar A, Coelho R, Rocco B, Palmer K. Dehydrated Human Amnion/Chorion Membrane Allograft Nerve Wrap Around the Prostatic 
Neurovascular Bundle Accelerates Early Return to Continence and Potency Following Robot-assisted Radical Prostatectomy: Propensity Score-matched Analysis. Eur Urol. 2015; 67: 977-80. doi: 10.1016/j.eururo.2015.01.012.

93. Tan G, Srivastave A, Grover S, Peters D, Dorsey P Jr, Scott A, Jhaveri J, Tilki D, Te A, Tewari A. Optimizing Vesicourethral Anastomosis Healing After Robot-Assisted Laparoscopic Radical Prostatectomy: Lessons Learned from Three Techniques in 1900 Patients. J Endourol. 2010; 24(12): 1975-83. doi: 10.1089/end.2009.0630.

94. Wiltz AL, Shikanov S, Eggener SE, Katz MH, Thong AE, Steinberg GD, Shalhav AL, Zagaja GP, Zorn KC. Robotic radical prostatectomy in overweight and obese patients: oncological and validated-functional outcomes. Urology. 2009; 73: 316-22. doi: 10.1016/j.urology.2008.08.493.

95. Froehner M KA, Koch R, Baretton GB, Hakenberg OW, Wirth MP. A combined index to classify prognostic comorbidity in candidates for radical prostatectomy. BMC Urol. 2014; 14: 28. doi: 10.1186/1471-2490-14-28.

96. Skolarus TA, Hedgepeth RC, Zhang Y, Weizer AZ, Montgomery JS, Miller DC, Wood DP, Jr., Hollenbeck BK. Does robotic technology mitigate the challenges of large prostate size? Urology. 2010; 76: 1117-21. doi: 10.1016/j. urology.2010.03.060.

97. Yasui T TK, Kurokawa S, Okada A, Mizuno K, Umemoto Y, Kawai N, Sasaki S, Hayashi Y, Kojima Y, Kohri K. Impact of prostate weight on perioperative outcomes of robot-assisted laparoscopic prostatectomy with a posterior approach to the seminal vesicle. BMC Urol. 2014; 14: 6 . doi: 10.1186/1471-2490-14-6.

98. Ficarra V, Novara G, Ahlering TE, Costello A, Eastham JA, Graefen M, Guazzoni G, Menon M, Mottrie A, Patel VR, Van der Poel H, Rosen RC, Tewari AK, et al. Systematic review and meta-analysis of studies reporting potency rates after robot-assisted radical prostatectomy. Eur Urol. 2012; 62: 418-30. doi: 10.1016/j.eururo.2012.05.046.

99. Jeong SJ, Yi J, Chung MS, Kim DS, Lee WK, Park H, Yoon CY, Hong SK, Byun SS, Lee SE. Early recovery of urinary continence after radical prostatectomy: correlation with vesico-urethral anastomosis location in the pelvic cavity measured by postoperative cystography. Int J Urol. 2011;
18: 444-51. doi: 10.1111/j.1442-2042.2011.02760.x.

100. Moran PS, O’Neill M, Teljeur C, Flattery M, Murphy LA, Smyth G, Ryan M. Robot-assisted radical prostatectomy compared with open and laparoscopic approaches: a systematic review and meta-analysis. Int J Urol. 2013; 20: 312-21. doi: 10.1111/iju.12070.

101. Robertson C, Close A, Fraser C, Gurung T, Jia X, Sharma P, Vale L, Ramsay C, Pickard R. Relative effectiveness of robot-assisted and standard laparoscopic prostatectomy as alternatives to open radical prostatectomy for treatment of localised prostate cancer: a systematic review and mixed treatment comparison meta-analysis. BJU Int. 2013; 112: 798-812. doi: 10.1111/bju.12247.

102. Liberati A, Altman DG, Tetzlaff J, Mulrow C, Gotzsche PC, Ioannidis JP, Clarke M, Devereaux PJ, Kleijnen J, Moher D. The PRISMA statement for reporting systematic reviews and meta-analyses of studies that evaluate health care interventions: explanation and elaboration. J Clin Epidemiol. 2009; 62: e1-34. doi: 10.1016/j. jclinepi.2009.06.006.

103. Dindo D, Demartines N, Clavien PA. Classification of Surgical Complications. Annals of Surgery. 2004; 240: 205 13. doi: 10.1097/01.sla.0000133083.54934.ae.

104. Clark HD, Wells GA, Huet C, McAlister FA, Salmi LR, Fergusson D, Laupacis A. Assessing the quality of randomized trials: reliability of the Jadad scale. Control Clin Trials. 1999; 20: 448-52. doi: 10.1016/s01972456(99)00026-4.

105. Taggart DP, D'Amico R, Altman DG. Effect of arterial revascularisation on survival: a systematic review of studies comparing bilateral and single internal mammary arteries. The Lancet. 2001; 358: 870-5. doi: 10.1016/s01406736(01)06069-x. 